\title{
The tau constant and the edge connectivity of a metrized graph
}

\author{
Zubeyir Cinkir * \\ Department of Mathematics, Faculty of Education \\ Zirve University, Gaziantep, TURKEY \\ zubeyir.cinkir@zirve.edu.tr
}

Submitted: Dec 17, 2011; Accepted: Nov 29, 2012; Published: Dec 13, 2012

Mathematics Subject Classification: 05C99, 94C99, 05C76

\begin{abstract}
The tau constant is an important invariant of a metrized graph. It has connections to other graph invariants such as Kirchhoff index, and it has applications to arithmetic properties of algebraic curves. We show how the tau constant of a metrized graph changes under successive edge contractions and deletions. We prove identities which we call "contraction", "deletion", and "contraction-deletion" identities on a metrized graph. By establishing a lower bound for the tau constant in terms of the edge connectivity, we prove that Baker and Rumely's lower bound conjecture on the tau constant holds for metrized graphs with edge connectivity 5 or more. We show that proving this conjecture for 3-regular graphs is enough to prove it for all graphs.
\end{abstract}

\section{Introduction}

The tau constant $\tau(\Gamma)$ is an invariant of a metrized graph $\Gamma$ which is a finite compact topological graph equipped with a distance function on its edges. One can find various foundational results concerning this invariant in [4] and [5]. Since a metrized graph has both analytic and combinatorial structure, the tau constant is analytic and combinatorial in nature, too. One can express [6, Theorem 1.1] $\tau(\Gamma)$ only in terms of the discrete Laplacian matrix of $\Gamma$ and its pseudo inverse.

Results of this paper are used in [7] to give an improved upper bound for the Kirchhoff index of $r$-regular graphs in terms of their edge connectivity. In another direction, applications of the results of this paper to arithmetic of algebraic curves and specifically

${ }^{*}$ I would like to thank Dr. Robert Rumely for his continued support and help. I also would like to thank to anonymous referee for very helpful and detailed feedback on earlier version of this paper.

THe ELECTRonic Journal of COMBinatorics 19(4) (2012), \#P46 
to the Effective Bogomolov Conjecture can be found in [8]. Among other things, in [8], we relate the tau constant to certain invariants of metrized graphs studied in [14].

Note that there is a 1-1 correspondence [3, Lemma 2.2] between equivalence classes of finite connected weighted graphs, metrized graphs, and resistive electric circuits. If an edge $e_{i}$ of a metrized graph has length $L_{i}$, then the resistance of $e_{i}$ is $L_{i}$ in the corresponding resistive electric circuit, and the weight of $e_{i}$ is $\frac{1}{L_{i}}$ in the corresponding weighted graph. Therefore, the identities we show in this paper have equivalent forms for weighted graphs.

The tau constant is a positive real-valued number associated to a given metrized graph (see $\S 2$ for more information). It was initially defined by M. Baker and R. Rumely [3, Theorem 14.1]. They [3] introduced a measure valued Laplacian operator $\Delta$ which extends Laplacian operators studied earlier in the articles [9] and [13]. This Laplacian operator combines the "discrete" Laplacian on a finite graph and the "continuous" Laplacian $-f^{\prime \prime}(x) d x$ on $\mathbb{R}$. Baker and Rumely [3] studied harmonic analysis on metrized graphs. In terms of spectral theory, the tau constant is the trace of the inverse operator of $\Delta$ when $\Gamma$ has total length 1 .

Baker and Rumely posed the following conjecture concerning a lower bound for $\tau(\Gamma)$.

Conjecture 1.1. [3] There is a universal constant $C>0$ such that for all metrized graphs $\Gamma, \tau(\Gamma) \geqslant C \cdot \ell(\Gamma)$ where $\ell(\Gamma)$ is the total length of $\Gamma$.

We call Conjecture 1.1 Baker and Rumely's lower bound conjecture. Equivalently, we can state Conjecture 1.1 as follows [5]:

Conjecture 1.2. If $\ell(\Gamma)=\int_{\Gamma} d x$ denotes the total length of $\Gamma$, then we have

$$
\inf _{\Gamma} \frac{\tau(\Gamma)}{\ell(\Gamma)}>0
$$

taking the infimum over all metrized graphs $\Gamma$ with $\ell(\Gamma) \neq 0$.

In summer 2003 at the University of Georgia, an REU group (REU at UGA, in short) lead by Baker and Rumely studied properties of the tau constant and the lower bound conjecture. We showed in [5] that this conjecture holds for various large classes of metrized graphs. Next, we briefly describe what we achieved in this paper aiming to prove the lower bound conjecture.

The first main result in this paper is the following theorem, which gives a lower bound for $\tau(\Gamma)$ in terms of the edge connectivity of $\Gamma$ when the edge connectivity is at least 4 (see Theorem 6.10).

Theorem 1.3. Let $\Gamma$ be a metrized graph with $v$ vertices, and let $\Lambda(\Gamma)$ be the edge connectivity of $\Gamma$. Then we have

$$
\begin{aligned}
& \text { 1. } \tau(\Gamma) \geqslant \ell(\Gamma)\left(\frac{1}{12}\left(1-\frac{4}{\Lambda(\Gamma)}\right)^{2}+\frac{4(\Lambda(\Gamma)-2)}{(v+6) \Lambda(\Gamma)^{2}}\right), \quad \text { if } \Lambda(\Gamma) \geqslant 4 \text {. In particular, } \tau(\Gamma) \geqslant \frac{\ell(\Gamma)}{108} \text { if } \\
& \Lambda(\Gamma) \geqslant 6, \text { and } \tau(\Gamma) \geqslant \frac{\ell(\Gamma)}{300} \text { if } \Lambda(\Gamma)=5 .
\end{aligned}
$$


2. $\tau(\Gamma) \geqslant \frac{\ell(\Gamma)}{2(v+6)}$. In particular, $\tau(\Gamma) \geqslant \frac{\ell(\Gamma)}{108}$ if $v \leqslant 48$.

Interestingly, Theorem 1.3 not only shows that Conjecture 1.1 holds for graphs with edge connectivity more than 4 , but also gives more refined information about the possible values of $\tau(\Gamma)$. The results of this theorem should be compared with the following upper bound: [5, Corollary 5.8 and Remark 2.15]:

$$
\tau(\Gamma) \leqslant \frac{\ell(\Gamma)}{12}, \quad \text { whenever } \Gamma \text { has edge connectivity at least two. }
$$

It follows from Theorem 1.3 and Equation (1) as an example that $\frac{27}{400} \ell(\Gamma)<\tau(\Gamma) \leqslant \frac{1}{12} \ell(\Gamma)$ for any graph with edge connectivity 40 and any edge lengths assignment.

The proof of Theorem 1.3 involves establishing a set of identities, which we call "contraction", "deletion", and "contraction-deletion" identities on a metrized graph (see $\S 4$ ). By using these identities, we show how the tau constant changes after successive edge deletions and contractions. In particular, when we consider successive edge contractions until we are left with only two vertices, we use our previous results [5] about the tau constant to obtain a set of inequalities between the terms adding up to the tau constant. In this way, we transform the tau lower bound problem into an optimization problem. The results here extend those obtained in [4, Sections 3.6, 3.7, 3.9, 3.10 and 3.12] in a coherent and systematic manner.

The second main result of this paper (see Theorem 7.2) is the following theorem:

Theorem 1.4. If there is a constant $C>0$ such that for all 3 -regular metrized graphs $\Gamma$, $\tau(\Gamma) \geqslant C \cdot \ell(\Gamma)$, then $\tau(\beta) \geqslant C \cdot \ell(\beta)$ for all metrized graphs $\beta$.

We think that Conjecture 1.1 can be refined as follows (see the last part of $\S 7$ ):

Conjecture 1.5. For all metrized graphs $\Gamma, \tau(\Gamma)>\frac{1}{108} \cdot \ell(\Gamma)$.

\section{Metrized graphs and their tau constants}

In this section, we recall a few facts about metrized graphs and their tau constants.

"Metrized graph" as a term was introduced by Rumely [12], and developed further in [9] and [13]. Here is a rigorous definition of a metrized graph:

Definition. [13][3, Definition 2.1] A metrized graph $\Gamma$ is a compact, connected metric space such that for each $p \in \Gamma$, there exist a radius $r_{p}>0$ and an integer $v(p) \geqslant 1$ such that $p$ has a neighborhood $V_{p}\left(r_{p}\right)$ isometric to the star-shaped set

$$
S\left(v(p), r_{p}\right)=\left\{z \in \mathbb{C}: z=t e^{k \cdot 2 \pi i / v(p)} \text { for some } 0 \leqslant t<r_{p} \text { and some } k \in \mathbb{Z}\right\},
$$

equipped with the path metric. 
A leisurely survey on metrized graphs can be found in [2]. Moreover, we have the following intuitional description of a metrized graph:

A metrized graph $\Gamma$ is a finite connected graph with a distinguished parametrization of each of its edges. $\Gamma$ can be considered as a one-dimensional manifold except at finitely many "branch points", where it looks locally like an $n$-pointed star. A metrized graph $\Gamma$ can have multiple edges and self-loops. Networks, metric graphs and quantum graphs are more or less the same as metrized graphs. We refer to [3, Section 1.9] to see various references for related work.

For any given $p \in \Gamma$, the number $v(p)$ of directions emanating from $p$ will be called the valence of $p$. By definition, there can be only finitely many $p \in \Gamma$ with $v(p) \neq 2$.

For a metrized graph $\Gamma$, we will denote a vertex set for $\Gamma$ by $V(\Gamma)$. We require that $V(\Gamma)$ be finite and non-empty and that $p \in V(\Gamma)$ for each $p \in \Gamma$ if $v(p) \neq 2$. For a given metrized graph $\Gamma$, it is possible to enlarge the vertex set $V(\Gamma)$ by considering additional valence 2 points as vertices.

For a given metrized graph $\Gamma$ with vertex set $V(\Gamma)$, the set of edges of $\Gamma$ is the set of closed line segments with end points in $V(\Gamma)$. We will denote the set of edges of $\Gamma$ by $E(\Gamma)$. However, if $e_{i}$ is an edge, by $\Gamma-e_{i}$ we mean the graph obtained by deleting the interior of $e_{i}$.

Let $v:=\#(V(\Gamma))$ and $e:=\#(E(\Gamma))$. We define the genus of $\Gamma$ to be the first Betti number $g(\Gamma):=e-v+1$ of the graph $\Gamma$. Note that the genus is a topological invariant of $\Gamma$. In particular, it is independent of the choice of the vertex set $V(\Gamma)$. Since $\Gamma$ is connected, $g(\Gamma)$ coincides with the cyclomatic number of $\Gamma$ in combinatorial graph theory. We will simply use $g$ to denote $g(\Gamma)$ when there is no danger of confusion. One should note that our definition of genus is different from the minimum integer $m$ for which the graph can be embedded into a compact connected 2-dimensional manifold homeomorphic to a sphere with $m$ handles. Our definition of genus is motivated by the fact that metrized graphs arise as dual graphs of special fibers of algebraic curves.

We denote the length of an edge $e_{i} \in E(\Gamma)$ by $L_{i}$, which represents a positive real number. The total length of $\Gamma$, which will be denoted by $\ell(\Gamma)$, is given by $\ell(\Gamma)=\sum_{i=1}^{e} L_{i}$.

Let $\Gamma$ be a metrized graph. If we scale each edge in $\Gamma$ by multiplying its length by $\frac{1}{\ell(\Gamma)}$, we obtain a new graph which is called the normalization of $\Gamma$ and denoted by $\Gamma^{N}$. Note that $\Gamma$ and $\Gamma^{N}$ have the same topology, and $\ell\left(\Gamma^{N}\right)=1$. If $\Gamma=\Gamma^{N}$, we call $\Gamma$ be a normalized graph.

A metrized graph $\Gamma$ is called $n$-regular if it has a vertex set $V(\Gamma)$ such that $v(p)=n$ for all vertices $p \in V(\Gamma)$.

We will denote the minimum of the valences of vertices in $V(\Gamma)$ by $\delta(\Gamma)$. The minimum number of edges whose deletion disconnects $\Gamma$ is called the "edge connectivity" of $\Gamma$ and denoted by $\Lambda(\Gamma)$. The minimum number of vertices whose deletion disconnects $\Gamma$ is called the "vertex connectivity" of $\Gamma$ and denoted by $\kappa(\Gamma)$.

In the article [9], a kernel $j_{x}(y, z)$ giving a fundamental solution of the Laplacian is defined and studied as a function of $x, y, z \in \Gamma$. For fixed $x$ and $z$ it has the following physical interpretation: When $\Gamma$ is viewed as a resistive electric circuit with terminals at $x$ and $z$, with the resistance in each edge given by its length, then $j_{x}(y, z)$ is the voltage 
difference between $y$ and $x$, when unit current enters at $z$ and exits at $x$ (with reference voltage 0 at $x$ ).

For any $x, y, z$ in $\Gamma$, the voltage function $j_{x}(y, z)$ on $\Gamma$ is a symmetric function in $y$ and $z$, and it satisfies $j_{x}(x, z)=0$ and $j_{x}(y, y)=r(x, y)$, where $r(x, y)$ is the resistance function on $\Gamma$. For each vertex set $V(\Gamma), j_{x}(y, z)$ is continuous on $\Gamma$ as a function of 3 variables. As the physical interpretation suggests, $j_{x}(y, z) \geqslant 0$ for all $x, y, z$ in $\Gamma$. For proofs of these facts, see the articles [9], [3, sec 1.5 and sec 6], and [13, Appendix]. The voltage function $j_{x}(y, z)$ and the resistance function $r(x, y)$ on a metrized graph were also studied in the articles [2] and [5].

The tau constant of a metrized graph was initially defined by Baker and Rumely [3, Section 14]. The following lemma gives a description of the tau constant. In particular, it implies that the tau constant is positive. We refer [5] and references cited therein for more information about the tau constant.

Lemma 2.1. [3, Lemma 14.4] For any fixed $y$ in $\Gamma, \tau(\Gamma)=\frac{1}{4} \int_{\Gamma}\left(\frac{\partial}{\partial x} r(x, y)\right)^{2} d x$.

We will use the following results frequently in later sections.

Lemma 2.2. [3, pg. 37] [5, Corollaries 2.17 and 2.22] If $\Gamma$ is a tree, i.e. a graph without cycles, then $\tau(\Gamma)=\frac{\ell(\Gamma)}{4}$. If $\Gamma$ is a circle graph, then $\tau(\Gamma)=\frac{\ell(\Gamma)}{12}$.

Remark 2.3. Whenever a graph $\Gamma$ has a vertex $p$ such that removing $p$ disconnects $\Gamma$, i.e. $p$ is a cut-vertex of $\Gamma$, then $\Gamma=\Gamma_{1} \cup \Gamma_{2}$ for subgraphs $\Gamma_{1}$ and $\Gamma_{2}$ with $\Gamma_{1} \cap \Gamma_{2}=\{p\}$. In this case, we have $\tau\left(\Gamma_{1} \cup \Gamma_{2}\right)=\tau\left(\Gamma_{1}\right)+\tau\left(\Gamma_{2}\right)$, which we call the additive property of the tau constant. It was initially noted in the REU at UGA.

Therefore, proving Conjecture 1.1 for graphs with vertex connectivity $\kappa(\Gamma) \geqslant 2$ yields it for all graphs.

Remark 2.4. [3] If we multiply all lengths on $\Gamma$ by a positive constant $c$, we obtain a graph $\Gamma^{\prime}$ of total length $c \cdot \ell(\Gamma)$. Then $\tau\left(\Gamma^{\prime}\right)=c \cdot \tau(\Gamma)$. This will be called the scale-independence of the tau constant. By this property, to prove Conjecture 1.1, it is enough to consider normalized metrized graphs.

Remark 2.5. For any metrized graph $\Gamma$, the tau constant $\tau(\Gamma)$ is independent of the vertex set $V(\Gamma)$ chosen. In particular, enlarging $V(\Gamma)$ by including points $p \in \Gamma$ with $v(p)=2$ does not change $\tau(\Gamma)$. Thus, $\tau(\Gamma)$ depends only on the topology and the edge length distribution of the metrized graph $\Gamma$. This will be called the valence property of the tau constant.

We will denote by $R_{i}(\Gamma)$, or by $R_{i}$ if there is no danger of confusion, the resistance between the end points of an edge $e_{i}$ of a graph $\Gamma$ when the interior of the edge $e_{i}$ is deleted from $\Gamma$. Recall that $R_{i}:=0$ if $e_{i}$ is a bridge. We will use the following notation in the rest of this paper:

$$
z(\Gamma)=\sum_{e_{i} \in E(\Gamma)} \frac{L_{i}^{2}}{L_{i}+R_{i}}, \quad r(\Gamma)=\sum_{e_{i} \in E(\Gamma)} \frac{L_{i} R_{i}}{L_{i}+R_{i}} .
$$


Note that $\ell(\Gamma)=z(\Gamma)+r(\Gamma)$.

Chinburg and Rumely [9, page 26] showed that

$$
\sum_{e_{i} \in E(\Gamma)} \frac{L_{i}}{L_{i}+R_{i}}=g, \quad \text { equivalently } \sum_{e_{i} \in E(\Gamma)} \frac{R_{i}}{L_{i}+R_{i}}=v-1 .
$$

Notation. Define $A_{p, q, \Gamma}:=\int_{\Gamma} j_{x}(p, q)\left(\frac{d}{d x} j_{p}(x, q)\right)^{2} d x$ for any $p, q \in \Gamma$.

Properties of $A_{p, q, \Gamma}$ were studied in the article [5, Sections 4 and 7]. For any $p, q \in \Gamma$, $0 \leqslant A_{p, q, \Gamma} \leqslant r(p, q)\left(r_{\Gamma}(p)-\frac{r(p, q)}{2}\right)$, where $r_{\Gamma}(p)=\max \{r(p, x) \mid x \in \Gamma\}$ and $r(x, y)$ is the resistance function in $\Gamma$. Here, the upper bound follows by combining [5, Theorem 4.3 part (vi)] and [5, Corollary 2.19].

We call an edge $e_{i} \in E(\Gamma)$ a bridge if $\Gamma-e_{i}$ is disconnected. If $\Gamma-e_{i}$ is connected for every $e_{i} \in E(\Gamma)$, we call $\Gamma$ a bridgeless graph.

Theorem 2.6. [5, Theorem 5.7] Let $\Gamma$ be a bridgeless graph. Suppose that $p_{i}$, $q_{i}$ are the end points of the edge $e_{i}$, for each $i=1,2, \ldots$, e. Then,

$$
\tau(\Gamma)=\frac{\ell(\Gamma)}{12}-\sum_{i=1}^{e} \frac{L_{i} A_{p_{i}, q_{i}, \Gamma-e_{i}}}{\left(L_{i}+R_{i}\right)^{2}}
$$

Theorem 2.7. [5, Theorem 2.21] For any $p, q \in \Gamma, \tau(\Gamma)=\frac{1}{4} \int_{\Gamma}\left(\frac{d}{d x} j_{x}(p, q)\right)^{2} d x+\frac{1}{4} r(p, q)$.

\section{$3 \quad$ Edge contractions and deletions}

Let $\bar{\Gamma}_{i}$ be the graph obtained by contracting the $i$-th edge $e_{i}, i \in\{1,2, \ldots, e\}$, of a given graph $\Gamma$ to its end points. If $e_{i} \in \Gamma$ has end points $p_{i}$ and $q_{i}$, then in $\bar{\Gamma}_{i}$, these points become identical, i.e., $p_{i}=q_{i}$. Let $\widetilde{\Gamma}_{i}$ be the graph obtained by identifying the end points of the $i$-th edge $e_{i} \in E(\Gamma)$. This makes $e_{i}$ into a loop in $\widetilde{\Gamma}_{i}$. Note that $\tau\left(\bar{\Gamma}_{i}\right)=\tau\left(\widetilde{\Gamma}_{i}\right)-\frac{L_{i}}{12}$ by the additive property of the tau constant and Lemma 2.2 .

The following lemma sheds light on how the tau constant changes by contraction of an edge:

Lemma 3.1. [5, Lemma 6.2] Let $e_{i} \in E(\Gamma)$ be such that $\Gamma-e_{i}$ is connected. Then we have

$$
\tau(\Gamma)=\tau\left(\bar{\Gamma}_{i}\right)+\frac{L_{i}}{12}-\frac{L_{i} A_{p_{i}, q_{i}, \Gamma-e_{i}}}{R_{i}\left(L_{i}+R_{i}\right)}
$$

Note that Lemma 3.1 involves terms containing $A_{p_{i}, q_{i}, \Gamma-e_{i}}$, which are fairly difficult to understand. One wants to understand the effect of edge contraction in a better way. An important step in this direction is provided by Proposition 3.2 and Theorem 3.3, which depend on Theorem 2.6.

Proposition 3.2. Let $\Gamma$ be a bridgeless graph with $v:=\#(V(\Gamma)) \geqslant 3$. Then,

$\tau(\Gamma)=\frac{1}{v-2} \sum_{i=1}^{e} \frac{R_{i}}{L_{i}+R_{i}} \tau\left(\bar{\Gamma}_{i}\right)-\frac{z(\Gamma)}{12(v-2)}, \quad \tau(\Gamma)=\frac{1}{v-2} \sum_{i=1}^{e} \frac{R_{i}}{L_{i}+R_{i}} \tau\left(\widetilde{\Gamma}_{i}\right)-\frac{\ell(\Gamma)}{12(v-2)}$. 
Proof. Multiply both sides of the equation in Lemma 3.1 by $\frac{R_{i}}{L_{i}+R_{i}}$, sum over all edges of $\Gamma$, and use the fact that $\sum_{i=1}^{e} \frac{R_{i}}{L_{i}+R_{i}}=v-1$ (see Equation (3)) to obtain

$$
(v-1) \tau(\Gamma)=\sum_{i=1}^{e} \frac{R_{i}}{L_{i}+R_{i}} \tau\left(\bar{\Gamma}_{i}\right)+\frac{r(\Gamma)}{12}-\sum_{i=1}^{e} \frac{L_{i} A_{p_{i}, q_{i}, \Gamma-e_{i}}}{\left(L_{i}+R_{i}\right)^{2}}
$$

Recall that $z(\Gamma)$ and $r(\Gamma)$ are defined in Equation (2). We obtain the first formula by using Theorem 2.6. Then the second formula follows from the fact that $\tau\left(\bar{\Gamma}_{i}\right)=\tau\left(\widetilde{\Gamma}_{i}\right)-\frac{L_{i}}{12}$.

In the proof of Proposition 3.2, we used the fact that $\Gamma$ is bridgeless when we worked with terms $A_{p_{i}, q_{i}, \Gamma-e_{i}}$. We will now extend the result of Proposition 3.2 to any connected graph $\Gamma$. For an edge $e_{i}$ which is a bridge in $\Gamma$, the end points $p_{i}$ and $q_{i}$ become disconnected in $\Gamma-e_{i}$, and so $R_{i}=\infty$. In such cases, if we use the limiting values of the corresponding terms, it is possible to extend Proposition 3.2 to a metrized graph with bridges. More precisely, note that

$$
\tau(\Gamma)=\frac{1}{v-2} \sum_{i=1}^{e}\left[\lim _{t \rightarrow R_{i}} \frac{t}{L_{i}+t}\right] \tau\left(\bar{\Gamma}_{i}\right)-\frac{1}{12(v-2)} \sum_{i=1}^{e}\left[\lim _{t \rightarrow R_{i}} \frac{L_{i}^{2}}{L_{i}+t}\right]
$$

In short, we set $\frac{R_{i}}{L_{i}+R_{i}}:=1$ and $\frac{L_{i}}{L_{i}+R_{i}}:=0$ whenever $R_{i}=\infty$.

Theorem 3.3. Let $\Gamma$ be a metrized graph with $v:=\#(V(\Gamma)) \geqslant 3$. Then we have

$\tau(\Gamma)=\frac{1}{v-2} \sum_{i=1}^{e} \frac{R_{i}}{L_{i}+R_{i}} \tau\left(\bar{\Gamma}_{i}\right)-\frac{z(\Gamma)}{12(v-2)}, \quad \tau(\Gamma)=\frac{1}{v-2} \sum_{i=1}^{e} \frac{R_{i}}{L_{i}+R_{i}} \tau\left(\widetilde{\Gamma}_{i}\right)-\frac{\ell(\Gamma)}{12(v-2)}$.

Proof. We already dealt with the case in which $\Gamma$ is bridgeless. Suppose that $\Gamma$ has bridges. Let $B=\left\{e_{i_{1}}, e_{i_{2}}, \ldots, e_{i_{k}}\right\}$ be the set of all bridges in $\Gamma$, for some positive integer $k$. Let $\gamma$ be the graph obtained from $\Gamma$ by contracting all of its bridges to their end points. Thus, an edge $e_{i}$ belongs to $E(\gamma)$ iff $e_{i} \notin B$. By the additive property of $\tau(\Gamma)$ (i.e., by Remark 2.3) and Lemma 2.2,

$$
\tau(\Gamma)=\tau(\gamma)+\frac{1}{4} \sum_{e_{j} \in B} L_{j}
$$

Clearly, $\gamma$ is connected and bridgeless with $v-k$ vertices. Note that if $e_{i} \in B$, then

$$
\tau\left(\bar{\Gamma}_{i}\right)=\tau(\gamma)+\frac{1}{4} \sum_{e_{j} \in B} L_{j}-\frac{L_{i}}{4}
$$

and if $e_{i} \notin B$, then

$$
\tau\left(\bar{\Gamma}_{i}\right)=\tau\left(\bar{\gamma}_{i}\right)+\frac{1}{4} \sum_{e_{j} \in B} L_{j}
$$


We have

$$
z(\Gamma)=z(\gamma), \text { and } \sum_{e_{i} \in E(\Gamma)-B} \frac{R_{i}(\Gamma)}{L_{i}+R_{i}(\Gamma)}=\sum_{e_{i} \in E(\gamma)} \frac{R_{i}(\gamma)}{L_{i}+R_{i}(\gamma)}=v-k-1
$$

Since $\gamma$ is bridgeless, we can apply Proposition 3.2 to obtain

$$
\tau(\gamma)=\frac{1}{v-k-2} \sum_{e_{i} \in E(\gamma)} \frac{R_{i}}{L_{i}+R_{i}} \tau\left(\bar{\gamma}_{i}\right)-\frac{z(\gamma)}{12(v-k-2)}
$$

Then by Equations (5) and (6)

$$
\begin{aligned}
\sum_{e_{i} \in E(\Gamma)} \frac{R_{i} \cdot \tau\left(\bar{\Gamma}_{i}\right)}{L_{i}+R_{i}}= & \sum_{e_{i} \notin B} \frac{R_{i}}{L_{i}+R_{i}}\left(\tau\left(\bar{\gamma}_{i}\right)+\sum_{e_{j} \in B} \frac{L_{j}}{4}\right)+\sum_{e_{i} \in B} \frac{R_{i}}{L_{i}+R_{i}}\left(\tau(\gamma)+\sum_{e_{j} \in B} \frac{L_{j}}{4}-\frac{L_{i}}{4}\right) \\
= & \sum_{e_{i} \notin B} \frac{R_{i}}{L_{i}+R_{i}} \tau\left(\bar{\gamma}_{i}\right)+\left(\frac{1}{4} \sum_{e_{j} \in B} L_{j}\right)\left(\sum_{e_{i} \notin B} \frac{R_{i}}{L_{i}+R_{i}}\right)+k \tau(\gamma) \\
& \quad+\frac{k-1}{4} \sum_{e_{j} \in B} L_{j}, \\
= & (v-2) \tau(\gamma)+\frac{z(\gamma)}{12}+\frac{v-2}{4} \sum_{e_{j} \in B} L_{j}, \quad \text { by Equations (8) and }(7) . \\
= & (v-2) \tau(\Gamma)+\frac{z(\Gamma)}{12}, \quad \text { by Equations (4) and (7). }
\end{aligned}
$$

This is equivalent to the first formula we wanted to show. By using the fact that $\tau\left(\widetilde{\Gamma}_{i}\right)=$ $\tau\left(\bar{\Gamma}_{i}\right)+\frac{L_{i}}{12}$, for all $e_{i} \in E(\Gamma)$, along with the first formula, we obtain the second formula.

The following lemma shows how the tau constant changes by deletion of an edge when the remaining graph is connected.

Lemma 3.4. [5, Corollary 5.3] Suppose that $\Gamma$ is a metrized graph such that $\Gamma-e_{i}$, for some edge $e_{i} \in E(\Gamma)$ with length $L_{i}$ and end points $p_{i}$ and $q_{i}$, is connected. Then we have

$$
\tau(\Gamma)=\tau\left(\Gamma-e_{i}\right)+\frac{L_{i}}{12}-\frac{R_{i}}{6}+\frac{A_{p_{i}, q_{i}, \Gamma-e_{i}}}{L_{i}+R_{i}} .
$$

We can combine Lemma 3.1 and Lemma 3.4 to obtain the following Lemma:

Lemma 3.5. Suppose that $\Gamma$ is a graph such that $\Gamma-e_{i}$, for some edge $e_{i} \in E(\Gamma)$ with length $L_{i}$ is connected. Then we have

$$
\tau(\Gamma)=\frac{L_{i}}{L_{i}+R_{i}} \tau\left(\Gamma-e_{i}\right)+\frac{R_{i}}{L_{i}+R_{i}} \tau\left(\bar{\Gamma}_{i}\right)+\frac{L_{i}^{2}-L_{i} R_{i}}{12\left(L_{i}+R_{i}\right)} .
$$

Proof. Multiply the formula in Lemma 3.1 by $\frac{R_{i}}{L_{i}+R_{i}}$ and the formula in Lemma 3.4 by $\frac{L_{i}}{L_{i}+R_{i}}$. Then add the results. 
To show the effect of edge deletion on the tau constant without using any terms with $A_{p_{i}, q_{i}, \Gamma-e_{i}}$, we have the following theorem:

Theorem 3.6. Let $\Gamma$ be a bridgeless graph with edges $\left\{e_{1}, e_{2}, \ldots, e_{e}\right\}$. Then,

$$
\tau(\Gamma)=\frac{1}{g+1} \sum_{i=1}^{e} \frac{L_{i}}{L_{i}+R_{i}} \tau\left(\Gamma-e_{i}\right)+\frac{\ell(\Gamma)}{6(g+1)}-\frac{r(\Gamma)}{4(g+1)}
$$

Proof. Multiply both sides of the equation given in Lemma 3.4 by $\frac{L_{i}}{L_{i}+R_{i}}$, sum over all edges of $\Gamma$, and use the fact that $\sum_{i=1}^{e} \frac{L_{i}}{L_{i}+R_{i}}=g$ (see Equation (3)) to obtain

$$
g \cdot \tau(\Gamma)=\sum_{i=1}^{e} \frac{L_{i}}{L_{i}+R_{i}} \tau\left(\Gamma-e_{i}\right)+\frac{z(\Gamma)}{12}-\frac{r(\Gamma)}{6}+\sum_{i=1}^{e} \frac{L_{i} A_{p_{i}, q_{i}, \Gamma-e_{i}}}{\left(L_{i}+R_{i}\right)^{2}}
$$

Finally, we use Theorem 2.6 and the fact that $z(\Gamma)+r(\Gamma)=\ell(\Gamma)$ to complete the proof.

As a corollary, we obtain a lower bound to the tau constant in terms of the genus $g$.

Corollary 3.7. Let $\Gamma$ be a bridgeless metrized graph. Let edge $e_{i}$ have end points $p_{i}$ and $q_{i}$. For the voltage function $j_{x}^{i}\left(p_{i}, q_{i}\right)$ on $\Gamma-e_{i}$, we have

$$
\tau(\Gamma)=\frac{1}{4(g+1)} \sum_{e_{i} \in E(\Gamma)} \frac{L_{i}}{L_{i}+R_{i}} \int_{\Gamma-e_{i}}\left(\frac{d}{d x} j_{x}^{i}\left(p_{i}, q_{i}\right)\right)^{2} d x+\frac{\ell(\Gamma)}{6(g+1)} .
$$

In particular, $\tau(\Gamma) \geqslant \frac{\ell(\Gamma)}{6(g+1)}$.

Proof. Applying Theorem 2.7 to $\Gamma-e_{i}$ gives $\tau\left(\Gamma-e_{i}\right)=\frac{1}{4} \int_{\Gamma-e_{i}}\left(\frac{d}{d x} j_{x}^{i}\left(p_{i}, q_{i}\right)\right)^{2} d x+\frac{R_{i}}{4}$ for any edge $e_{i}$. Thus, we obtain what we want by substituting this into Theorem 3.6.

The results given in Theorem 3.3 and Theorem 3.6 allow us to compare $\tau(\Gamma)$ with $\tau\left(\bar{\Gamma}_{i}\right), \tau\left(\widetilde{\Gamma}_{i}\right)$ and $\tau\left(\Gamma-e_{i}\right)$. This suggests that one may apply induction arguments on the number of edges or vertices of $\Gamma$ to prove Conjecture 1.1. We attempted to follow this strategy without a satisfactory outcome. Based on our computations, we believe that we need further improvements on the results about $A_{p_{i}, q_{i}, \Gamma-e_{i}}$ to make the induction arguments applicable.

\section{Contraction and deletion identities}

In this section, we will prove a number of identities, which we call "contraction identities", "deletion identities" and "contraction-deletion" identities. These identities are interesting in their own right. One way to relate these identities to the tau constant can be explained as follows: 
We know the exact values of the tau constant when the metrized graph is a tree or circle (see Lemma 2.2). If a metrized graph has vertex connectivity 1 or 2 , we can express its tau constant in terms of the tau constants of its subgraphs (see Remark 2.3 and [5, Theorems 5.1 and 7.3]). After a sequence of edge deletions and contractions we can pass to these type of graphs from an arbitrary metrized graph. In the previous section, we gave formulas expressing $\tau(\Gamma)$ in terms of $\tau\left(\Gamma-e_{i}\right)$ 's or $\tau\left(\bar{\Gamma}_{i}\right)$ 's by considering all edge deletions or contractions of depth 1 (see Theorem 3.3 and Theorem 3.6). One wonders if it is possible to generalize these two theorems with further depths of edge deletions and contractions. The solution is given by the identities shown in this section. The identities of this section have crucial roles in generalizing the results of the previous section, as in the following section where we deal with the successive edge contractions.

Some of these "contraction identities", "deletion identities" and "contraction-deletion" identities were proven in [4, Sections 3.6 and 3.7] using different methods. Our approach in this paper is to utilize Euler's formula for homogeneous functions as in the proof of Theorem 2.6 in [5, pg 35].

Let $\Gamma$ be a graph with edges $E(\Gamma)=\left\{e_{1}, e_{2}, \ldots, e_{e}\right\}$, and let $\Gamma-e_{i}$ be the graph obtained by deleting the $i$-th edge $e_{i} \in E(\Gamma)$. As before $L_{i}$ is the length of edge $e_{i}$. Let $\Gamma^{D A}$ be the graph obtained from $\Gamma$ by replacing each edge $e_{i} \in E(\Gamma)$ by 2 edges $e_{i, 1}, e_{i, 2}$ of equal lengths $\frac{L_{i}}{2}$. Here DA stands for "Double Adjusted". Then, $V(\Gamma)=V\left(\Gamma^{D A}\right)$ and $\ell(\Gamma)=\ell\left(\Gamma^{D A}\right)$.

Given a graph $\Gamma$, we will compare $\tau$-constants of the following graphs: $\Gamma, \Gamma-e_{i}, \Gamma^{D A}$, $\left(\Gamma-e_{i}\right)^{D A}, \Gamma^{D A}-e_{i, j}$ and $\Gamma^{D A}-\left\{e_{i, 1}, e_{i, 2}\right\}$. It will turn out that by doing so, we will obtain non-trivial identities for $\tau(\Gamma), z(\Gamma)$ and $r(\Gamma)$.

The graphs in Figure 1 illustrate what we will do. Graph $I$ shows $\Gamma$ with an edge $e_{i} \in E(\Gamma)$. II shows $\Gamma-e_{i}, I I I$ shows $\left(\Gamma-e_{i}\right)^{D A}, I V$ shows $\Gamma^{D A}$ with edges $e_{i, 1}$ and $e_{i, 2}$, $V$ shows $\Gamma^{D A}-e_{i, 2}$ and $V I$ shows $\Gamma^{D A}-\left\{e_{i, 1}, e_{i, 2}\right\}$.

Note that $\left(\Gamma-e_{i}\right)^{D A}$ and $\Gamma^{d_{i}}:=\Gamma^{D A}-\left\{e_{i, 1}, e_{i, 2}\right\}$ are the same graphs. In Figure 1, they are the graphs in $I I I$ and $V I$.

Theorem 4.1. Let $\Gamma$ be a metrized graph, and let $e_{i} \in E(\Gamma)$ with end points $p_{i}$ and $q_{i}$. If $\Gamma-e_{i}$ is connected, we have

$$
\tau\left(\Gamma^{D A}\right)=\tau\left(\left(\Gamma-e_{i}\right)^{D A}\right)+\frac{2 L_{i}^{2}-R_{i}^{2}}{24\left(L_{i}+R_{i}\right)}+\frac{4}{L_{i}+R_{i}} A_{p_{i}, q_{i}, \Gamma^{d_{i}}} .
$$

Proof. By applying Lemma 3.4 to $\Gamma^{D A}$ for the edge $e_{i, 1} \in E\left(\Gamma^{D A}\right)$ and using $R_{i, 1}\left(\Gamma^{D A}\right)=$ $\frac{1}{2} \frac{L_{i} R_{i}}{2 L_{i}+R_{i}}$ from [5, Lemma 3.10 with $\left.n=2\right]$, we get

$$
\tau\left(\Gamma^{D A}\right)=\tau\left(\Gamma^{D A}-e_{i, 1}\right)+\frac{L_{i}}{24}-\frac{1}{12} \frac{L_{i} R_{i}}{2 L_{i}+R_{i}}+\frac{A_{p_{i}, q_{i}, \Gamma^{D A}-e_{i, 1}}}{\frac{L_{i}}{2}+\frac{1}{2} \frac{L_{i} R_{i}}{2 L_{i}+R_{i}}} .
$$

By applying $[5$, Lemma 7.5$]$ to $A_{p_{i}, q_{i}, \Gamma^{D A}-e_{i, 1}}$ with edge $e_{i, 2}$, we obtain

$$
A_{p_{i}, q_{i}, \Gamma^{D A}-e_{i, 1}}=\frac{4 L_{i}^{2} A_{p_{i}, q_{i}, \Gamma^{d_{i}}}}{\left(2 L_{i}+R_{i}\right)^{2}}+\frac{1}{24}\left(\frac{L_{i} R_{i}}{2 L_{i}+R_{i}}\right)^{2}
$$




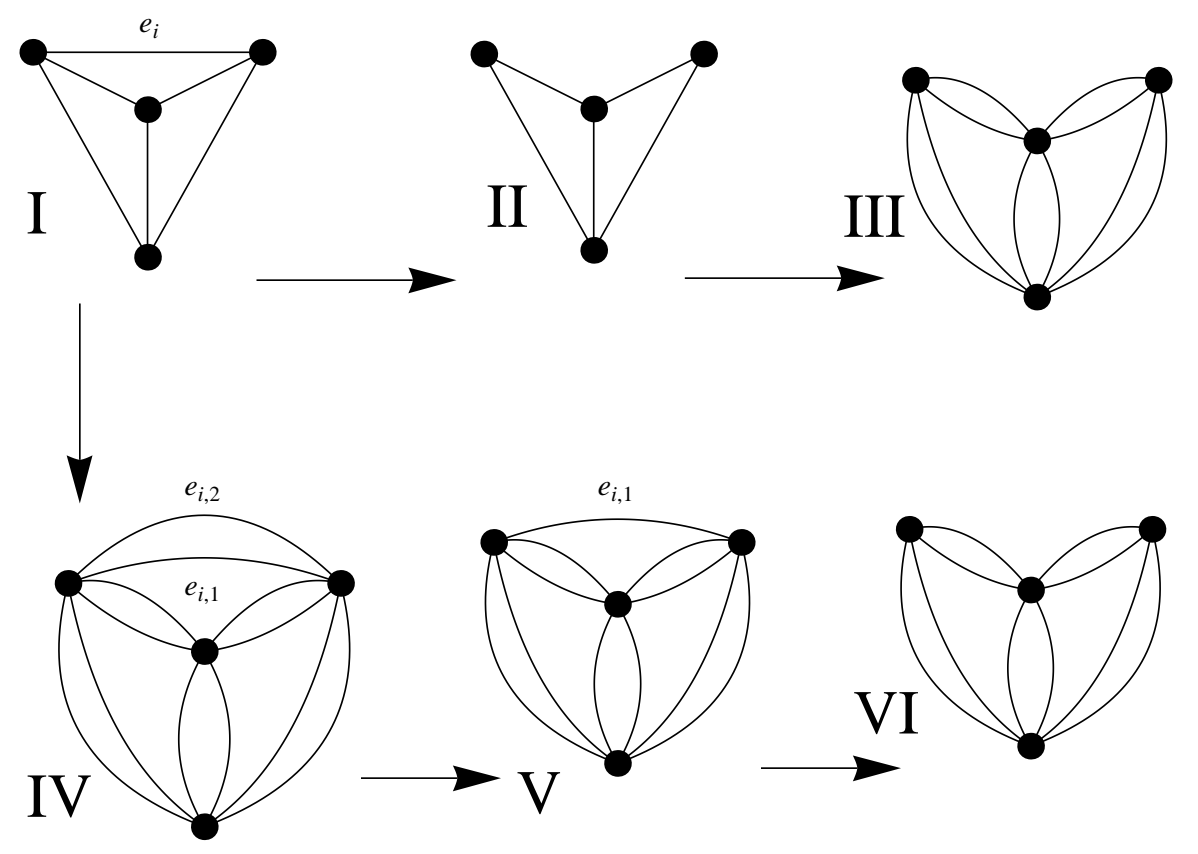

Figure 1: Graphs used to obtain the deletion identities.

Next, applying Lemma 3.4 to $\Gamma^{D A}-e_{i, 1}$ with respect to the edge $e_{i, 2}$ and using $R_{i, 2}\left(\Gamma^{D A}-\right.$ $\left.e_{i, 1}\right)=\frac{R_{i}}{4}$ gives

$$
\tau\left(\Gamma^{D A}-e_{i, 1}\right)=\tau\left(\Gamma^{d_{i}}\right)+\frac{L_{i}}{24}-\frac{R_{i}}{24}+\frac{4 A_{p_{i}, q_{i}, \Gamma^{d_{i}}}}{2 L_{i}+R_{i}} .
$$

Since $\Gamma^{d_{i}}=\left(\Gamma-e_{i}\right)^{D A}$, substituting Equations (10) and (11) into Equation (9) gives the result.

Notation. Let $\Gamma$ be a metrized graph. For each edge $e_{i} \in E(\Gamma)$ such that $\Gamma-e_{i}$ is connected, we set

$$
K_{i}(\Gamma):=\sum_{\substack{e_{j} \in E(\Gamma) \\ j \neq i}} \frac{L_{j}^{2}}{L_{j}+R_{j}}-\sum_{e_{j} \in E\left(\Gamma-e_{i}\right)} \frac{L_{j}^{2}}{L_{j}+R_{j}\left(\Gamma-e_{i}\right)} .
$$

Remark 4.2. Let $\Gamma$ be a metrized graph and let $e_{i} \in E(\Gamma)$. For every $j \neq i$ and $j \in$ $\{1,2, \ldots, e\}, R_{j}\left(\Gamma-e_{i}\right) \geqslant R_{j}$ by Rayleigh's Cutting law, which states that cutting branches can only increase the effective resistance between any two points in a circuit (See [10] for more information). Therefore, $\frac{L_{j}^{2}}{L_{j}+R_{j}\left(\Gamma-e_{i}\right)} \leqslant \frac{L_{j}^{2}}{L_{j}+R_{j}}$. Hence, $K_{i}(\Gamma) \geqslant 0$.

Theorem 4.3. Let $\Gamma$ be a metrized graph, and let $e_{i} \in E(\Gamma)$ with end points $p_{i}$ and $q_{i}$. If $\Gamma-e_{i}$ is connected, we have

$$
\frac{A_{p_{i}, q_{i}, \Gamma-e_{i}}}{L_{i}+R_{i}}=\frac{16 A_{p_{i}, q_{i}, \Gamma^{d_{i}}}}{L_{i}+R_{i}}-\frac{K_{i}(\Gamma)}{6} .
$$


Proof. Note that $\ell\left(\Gamma^{D A}\right)=\ell(\Gamma)$. Applying [5, Corollary 3.5] to $\Gamma^{D A}$, we obtain

$$
\tau\left(\Gamma^{D A}\right)=\frac{\ell(\Gamma)}{48}+\frac{\tau(\Gamma)}{4}+\frac{z(\Gamma)}{24} .
$$

Applying [5, Corollary 3.5] to $\left(\Gamma-e_{i}\right)^{D A}$, we obtain

$$
\tau\left(\left(\Gamma-e_{i}\right)^{D A}\right)=\frac{\ell\left(\Gamma-e_{i}\right)}{48}+\frac{\tau\left(\Gamma-e_{i}\right)}{4}+\frac{z\left(\Gamma-e_{i}\right)}{24} .
$$

Substituting Equation (12) and Equation (13) into Theorem 4.1, and recalling that $\ell(\Gamma-$ $\left.e_{i}\right)=\ell(\Gamma)-L_{i}$ gives

$$
\tau(\Gamma)=\tau\left(\Gamma-e_{i}\right)+\frac{L_{i}}{12}-\frac{R_{i}}{6}-\frac{K_{i}(\Gamma)}{6}+\frac{16 A_{p_{i}, q_{i}, \Gamma^{d}}}{L_{i}+R_{i}} .
$$

Comparing Equation (14) with Lemma 3.4 gives the result.

Let $p, q$ be any two points in $\Gamma$, and let $e_{0}$ be a line segment of length $L$. By identifying the end points of $e_{0}$ with $p$ and $q$ of $\Gamma$ we obtain a new graph which we denote by $\Gamma_{(p, q)}$. Then $\ell\left(\Gamma_{(p, q)}\right)=\ell(\Gamma)+L$. Also, by identifying $p$ and $q$ with each other in $\Gamma$ we obtain a graph which we denote by $\Gamma_{p q}$. Then $\ell\left(\Gamma_{p q}\right)=\ell(\Gamma)$. If $p$ and $q$ are end points of an edge $e_{i} \in \Gamma$, then $\Gamma_{p q}=\widetilde{\Gamma}_{i}$. The following corollary is the initial step towards the contraction-deletion identities (Theorems 4.9 and 4.5).

Corollary 4.4. Let $\Gamma$ be a metrized graph with resistance function $r(x, y)$, and let $p, q$, $e_{0}$ and $\Gamma_{(p, q)}$ be as above. Corresponding to the edge $e_{0}$, suppose that we have the pair of edges $e_{0,1}$ and $e_{0,2}$ in $E\left(\left(\Gamma_{(p, q)}\right)^{D A}\right)$. Then we have

$$
\frac{A_{p, q, \Gamma}}{L+r(p, q)}=\frac{16 A_{p, q, \Gamma^{D A}}}{L+r(p, q)}-\frac{1}{6}\left(\sum_{\substack{e_{j} \in E\left(\Gamma_{(p, q)}\right) \\ e_{j} \neq e_{0}}} \frac{L_{j}^{2}}{L_{j}+R_{j}\left(\Gamma_{(p, q)}\right)}-\sum_{e_{i} \in E(\Gamma)} \frac{L_{i}^{2}}{L_{i}+R_{i}}\right) .
$$

Proof. Theorem 4.3 applied to $\Gamma_{(p, q)}$ with edge $e_{0}$ gives

$$
\begin{aligned}
& \frac{A_{p, q, \Gamma_{(p, q)}-e_{0}}}{L+r(p, q)}=\frac{16 A_{p, q,\left(\Gamma_{(p, q)}\right)^{D A}-\left\{e_{0,1}, e_{0,2}\right\}}}{L+r(p, q)}-\frac{1}{6} \sum_{\substack{e_{j} \in E\left(\Gamma_{(p, q)}\right) \\
e_{j} \neq e_{0}}} \frac{L_{j}^{2}}{L_{j}+R_{j}\left(\Gamma_{(p, q)}\right)} \\
& +\frac{1}{6} \sum_{e_{i} \in E\left(\Gamma_{(p, q)}-e_{0}\right)} \frac{L_{i}^{2}}{L_{i}+R_{i}}
\end{aligned}
$$

On the other hand, we have $\Gamma_{(p, q)}-e_{0}=\Gamma$ and $\left(\Gamma_{(p, q)}\right)^{D A}-\left\{e_{0,1}, e_{0,2}\right\}=\Gamma^{D A}$. This gives the result. 
Let $\Gamma_{(p, q)}^{\prime}$ be a metrized graph obtained from $\Gamma$ by connecting the points $p$ and $q$ of $\Gamma$ with line segment $e_{0}^{\prime}$ of length $L^{\prime}$. Then, $\Gamma_{(p, q)}-e_{0}=\Gamma_{(p, q)}^{\prime}-e_{0}^{\prime}$. Let $L=t_{1} \cdot r(p, q)$ and $L^{\prime}=t_{2} \cdot r(p, q)$ for some positive real numbers $t_{1}$ and $t_{2}$. By applying Corollary 4.4 to $\Gamma_{(p, q)}$ and $\Gamma_{(p, q)}^{\prime}$, we obtain

$$
\left(1+t_{1}\right)\left(z\left(\Gamma_{(p, q)}\right)-\frac{L^{2}}{L+r(p, q)}-z(\Gamma)\right)=\left(1+t_{2}\right)\left(z\left(\Gamma_{(p, q)}^{\prime}\right)-\frac{\left(L^{\prime}\right)^{2}}{L^{\prime}+r(p, q)}-z(\Gamma)\right) .
$$

As $t_{2} \rightarrow 0$, we have $L^{\prime} \rightarrow 0$ and $\Gamma_{(p, q)}^{\prime} \rightarrow \Gamma_{p q}$, and so $z\left(\Gamma_{(p, q)}^{\prime}\right) \rightarrow z\left(\Gamma_{p q}\right)$. We substitute $t_{1}=\frac{L}{r(p, q)}$ into Equation (15). Then we obtain the following relation as $t_{2} \rightarrow 0$ :

$$
z\left(\Gamma_{(p, q)}\right)=\frac{L^{2}}{L+r(p, q)}+\frac{L}{L+r(p, q)} z(\Gamma)+\frac{r(p, q)}{L+r(p, q)} z\left(\Gamma_{p q}\right) .
$$

We use Equation (16) to obtain the following Theorem:

Theorem 4.5. Let $\Gamma$ be a metrized graph. For each edge $e_{i} \in E(\Gamma)$ such that $\Gamma-e_{i}$ is connected, we have

$$
z(\Gamma)=\frac{L_{i}^{2}}{L_{i}+R_{i}}+\frac{L_{i}}{L_{i}+R_{i}} z\left(\Gamma-e_{i}\right)+\frac{R_{i}}{L_{i}+R_{i}} z\left(\bar{\Gamma}_{i}\right) .
$$

Proof. In Equation (16), replace $\Gamma_{(p, q)}$ by $\Gamma, L$ by $L_{i}, \Gamma$ by $\Gamma-e_{i}$. This gives what we wanted to show.

We call the identity in Theorem 4.5 the contraction-deletion identity for $z(\Gamma)$.

If $e_{i}$ is a bridge (i.e., $\left.R_{i}=\infty\right), z(\Gamma)=z\left(\bar{\Gamma}_{i}\right)$, which can also be seen from Theorem 4.5 as $R_{i} \rightarrow \infty$.

Moreover, for any metrized graph $\Gamma$ and for each edge $e_{i} \in E(\Gamma)$ such that $\Gamma-$ $e_{i}$ is connected, we obtain the expression below for $K_{i}(\Gamma)$ by using its definition and Theorem 4.5:

$$
K_{i}(\Gamma)=\frac{R_{i}}{L_{i}+R_{i}}\left(z\left(\bar{\Gamma}_{i}\right)-z\left(\Gamma-e_{i}\right)\right) .
$$

A function $f: \mathbb{R}^{n} \rightarrow \mathbb{R}$ is called homogeneous of degree $k$ if

$$
f\left(\lambda x_{1}, \lambda x_{2}, \ldots, \lambda x_{n}\right)=\lambda^{k} f\left(x_{1}, x_{2}, \ldots, x_{n}\right), \text { for } \lambda>0 .
$$

A continuously differentiable function $f: \mathbb{R}^{n} \rightarrow \mathbb{R}$ which is homogeneous of degree $k$ has the following property:

$$
k \cdot f=\sum_{i=1}^{n} x_{i} \frac{\partial f}{\partial x_{i}} .
$$

Equation (18) is called Euler's formula.

For a given metrized graph $\Gamma$ with $\#(E(\Gamma))=e$, let $\left\{L_{1}, L_{2}, \ldots, L_{e}\right\}$ be the edge lengths. Then $z: \mathbb{R}_{>0}^{e} \rightarrow \mathbb{R}$ given by $z\left(L_{1}, L_{2}, \ldots, L_{e}\right)=z(\Gamma)$ is a continuously differentiable homogeneous function of degree 1 , when we consider all possible length distributions without changing the topology of the graph $\Gamma$. 
Lemma 4.6. Let $\Gamma$ be a metrized graph, and let $e_{i} \in E(\Gamma)$ be an edge of length $L_{i}$ such that $\Gamma-e_{i}$ is connected. Then we have

$$
\frac{\partial z(\Gamma)}{\partial L_{i}}=\frac{L_{i}\left(L_{i}+2 R_{i}\right)}{\left(L_{i}+R_{i}\right)^{2}}+\frac{R_{i}}{\left(L_{i}+R_{i}\right)^{2}} z\left(\Gamma-e_{i}\right)-\frac{R_{i}}{\left(L_{i}+R_{i}\right)^{2}} z\left(\bar{\Gamma}_{i}\right) .
$$

Proof. Note that $z\left(\bar{\Gamma}_{i}\right), z\left(\Gamma-e_{i}\right)$ and $R_{i}$ are independent of $L_{i}$. Thus, taking the partial derivatives of the both sides of the identity in Theorem 4.5 with respect to $L_{i}$ gives the result.

Theorem 4.7. Let $\Gamma$ be a bridgeless metrized graph. Then we have

$$
\sum_{e_{i} \in E(\Gamma)} \frac{L_{i} K_{i}(\Gamma)}{L_{i}+R_{i}}=\sum_{e_{i} \in E(\Gamma)} \frac{L_{i} R_{i}}{\left(L_{i}+R_{i}\right)^{2}}\left(z\left(\bar{\Gamma}_{i}\right)-z\left(\Gamma-e_{i}\right)\right)=\sum_{e_{i} \in E(\Gamma)} \frac{L_{i}^{2} R_{i}}{\left(L_{i}+R_{i}\right)^{2}} .
$$

Proof. The first equality follows from Equation (17). By Euler's formula,

$$
z(\Gamma)=\sum_{e_{i} \in E(\Gamma)} L_{i} \cdot \frac{\partial z(\Gamma)}{\partial L_{i}}
$$

Then the second equality follows from Lemma 4.6.

For a given metrized graph $\Gamma$ with $\#(E(\Gamma))=e$, let $\left\{L_{1}, L_{2}, \ldots, L_{e}\right\}$ be the edge lengths. Then $r: \mathbb{R}_{>0}^{e} \rightarrow \mathbb{R}$ given by $r\left(L_{1}, L_{2}, \ldots, L_{e}\right)=r(\Gamma)$ is a continuously differentiable homogeneous function of degree 1 , when we consider all possible length distributions without changing the topology of the graph $\Gamma$.

Lemma 4.8. Let $\Gamma$ be a metrized graph, and let $e_{i} \in E(\Gamma)$ be an edge of length $L_{i}$ such that $\Gamma-e_{i}$ is connected. Then we have

$$
\frac{\partial r(\Gamma)}{\partial L_{i}}=\frac{R_{i}^{2}}{\left(L_{i}+R_{i}\right)^{2}}+\frac{R_{i}}{\left(L_{i}+R_{i}\right)^{2}} r\left(\Gamma-e_{i}\right)-\frac{R_{i}}{\left(L_{i}+R_{i}\right)^{2}} r\left(\bar{\Gamma}_{i}\right) .
$$

Proof. Since $\ell(\Gamma)=z(\Gamma)+r(\Gamma)$ for any graph, and $\ell\left(\Gamma-e_{i}\right)=\ell\left(\bar{\Gamma}_{i}\right)=\ell(\Gamma)-L_{i}$, Theorem 4.5 is equivalent to

$$
r(\Gamma)=\frac{L_{i} R_{i}}{L_{i}+R_{i}}+\frac{L_{i}}{L_{i}+R_{i}} r\left(\Gamma-e_{i}\right)+\frac{R_{i}}{L_{i}+R_{i}} r\left(\bar{\Gamma}_{i}\right) .
$$

Note that $r\left(\bar{\Gamma}_{i}\right), r\left(\Gamma-e_{i}\right)$ and $R_{i}$ are independent of $L_{i}$. Thus, taking the partial derivatives of the both sides of Equation (19) with respect to $L_{i}$ gives the result.

Let $\Gamma-e_{i}$ be a connected graph for an edge $e_{i} \in E(\Gamma)$ of length $L_{i}$. Suppose $p_{i}$ and $q_{i}$ are the end points of $e_{i}$, and $p \in \Gamma-e_{i}$. By applying circuit reductions, $\Gamma-e_{i}$ can be transformed into a $Y$-shaped graph with the same resistances between $p_{i}, q_{i}$, and $p$ as in $\Gamma-e_{i}$ (see the articles [9] and [5, Section 2]). The resulting graph is shown by the first graph in Figure 2, with the corresponding voltage values on each segment, where 

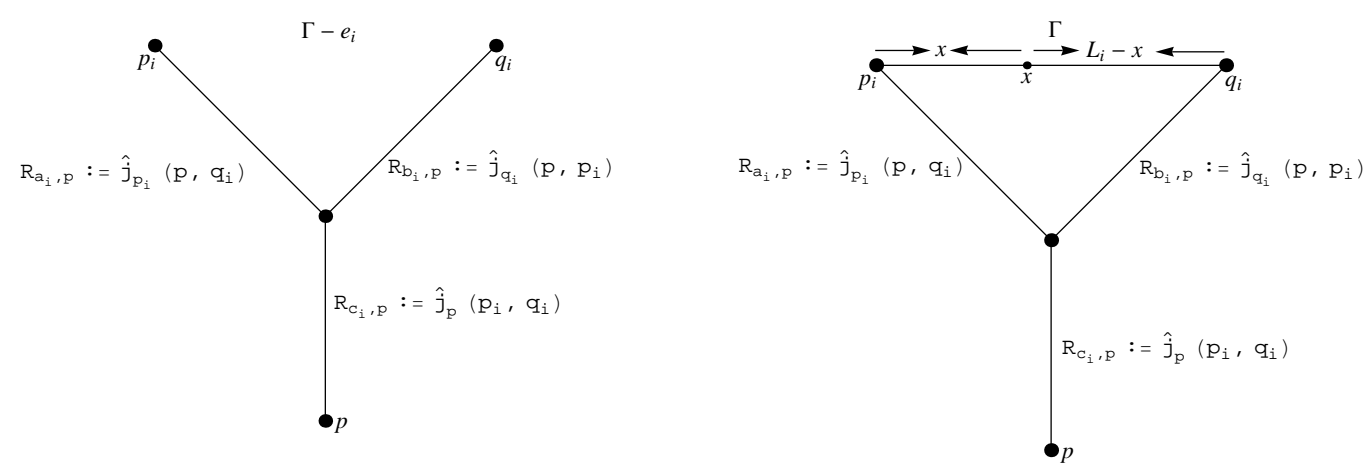

Figure 2: Circuit reduction of $\Gamma-e_{i}$ with reference to $p_{i}, q_{i}$ and $p$.

$\hat{j}_{x}(y, z)$ is the voltage function in $\Gamma-e_{i}$. Since $\Gamma-e_{i}$ has such circuit reduction, $\Gamma$ has the circuit reduction as the second graph in Figure 2. From now on, we will use the following notation: $R_{a_{i}, p}:=\hat{j}_{p_{i}}\left(p, q_{i}\right), R_{b_{i}, p}:=\hat{j}_{q_{i}}\left(p_{i}, p\right), R_{c_{i}, p}:=\hat{j}_{p}\left(p_{i}, q_{i}\right)$. Let $R_{i}$ be the resistance between $p_{i}$ and $q_{i}$ in $\Gamma-e_{i}$. Note that $R_{a_{i}, p}+R_{b_{i}, p}=R_{i}$ for each $p \in \Gamma$.

If $\Gamma-e_{i}$ is not connected, we set $R_{b_{i}, p}=R_{i}=\infty$ and $R_{a_{i}, p}=0$ if $p$ belongs to the component of $\Gamma-e_{i}$ containing $p_{i}$, and we set $R_{a_{i}, p}=R_{i}=\infty$ and $R_{b_{i}, p}=0$ if $p$ belongs to the component of $\Gamma-e_{i}$ containing $q_{i}$.

In the rest of the paper, for any metrized graph $\Gamma$ and a fixed vertex $p \in V(\Gamma)$ we will use the following notation:

$$
\begin{aligned}
& y(\Gamma)=\frac{1}{4} \sum_{e_{i} \in E(\Gamma)} \frac{L_{i} R_{i}^{2}}{\left(L_{i}+R_{i}\right)^{2}}+\frac{3}{4} \sum_{e_{i} \in E(\Gamma)} \frac{L_{i}\left(R_{a_{i}, p}-R_{b_{i}, p}\right)^{2}}{\left(L_{i}+R_{i}\right)^{2}}, \\
& x(\Gamma)=\sum_{e_{i} \in E(\Gamma)} \frac{L_{i}^{2} R_{i}}{\left(L_{i}+R_{i}\right)^{2}}+\frac{3}{4} \sum_{e_{i} \in E(\Gamma)} \frac{L_{i} R_{i}^{2}}{\left(L_{i}+R_{i}\right)^{2}}-\frac{3}{4} \sum_{e_{i} \in E(\Gamma)} \frac{L_{i}\left(R_{a_{i}, p}-R_{b_{i}, p}\right)^{2}}{\left(L_{i}+R_{i}\right)^{2}} .
\end{aligned}
$$

Note that $x(\Gamma)$ and $y(\Gamma)$ do not depend on the choice of vertices $p$ [5, Lemma 2.11]. If $\Gamma-e_{i}$ is not connected for an edge $e_{i}$, i.e. $R_{i}$ is infinite (and $\left.\left(R_{a_{i}, p}-R_{b_{i}, p}\right)^{2}=R_{i}^{2}\right)$, the summands should be considered as their corresponding limits as $R_{i} \longrightarrow \infty$.

It follows from [5, Proposition 2.9] that

$$
\tau(\Gamma)=\frac{\ell(\Gamma)}{12}-\frac{x(\Gamma)}{6}+\frac{y(\Gamma)}{6} .
$$

It is easy to see that

$$
r(\Gamma)=x(\Gamma)+y(\Gamma), \quad \text { and so } \quad \ell(\Gamma)=x(\Gamma)+y(\Gamma)+z(\Gamma) .
$$

We call the following identities the contraction-deletion identities for $x(\Gamma)$ and $y(\Gamma)$. 
Theorem 4.9. Let $\Gamma$ be a metrized graph with an edge $e_{i} \in E(\Gamma)$ such that $\Gamma-e_{i}$ is connected. Then we have

$$
\begin{aligned}
& x(\Gamma)=\frac{L_{i} R_{i}}{L_{i}+R_{i}}+\frac{L_{i}}{L_{i}+R_{i}} x\left(\Gamma-e_{i}\right)+\frac{R_{i}}{L_{i}+R_{i}} x\left(\bar{\Gamma}_{i}\right), \\
& y(\Gamma)=\frac{L_{i}}{L_{i}+R_{i}} y\left(\Gamma-e_{i}\right)+\frac{R_{i}}{L_{i}+R_{i}} y\left(\bar{\Gamma}_{i}\right) .
\end{aligned}
$$

Proof. By Equations (19) and (21),

$$
x(\Gamma)+y(\Gamma)=\frac{L_{i} R_{i}}{L_{i}+R_{i}}+\frac{L_{i}}{L_{i}+R_{i}}\left(x\left(\Gamma-e_{i}\right)+y\left(\Gamma-e_{i}\right)\right)+\frac{R_{i}}{L_{i}+R_{i}}\left(x\left(\bar{\Gamma}_{i}\right)+y\left(\bar{\Gamma}_{i}\right)\right) .
$$

On the other hand, by Lemma 3.5 and Equation (20) applied to each of $\Gamma, \Gamma-e_{i}$ and $\bar{\Gamma}_{i}$ we have

$$
x(\Gamma)-y(\Gamma)=\frac{L_{i} R_{i}}{L_{i}+R_{i}}+\frac{L_{i}}{L_{i}+R_{i}}\left(x\left(\Gamma-e_{i}\right)-y\left(\Gamma-e_{i}\right)\right)+\frac{R_{i}}{L_{i}+R_{i}}\left(x\left(\bar{\Gamma}_{i}\right)-y\left(\bar{\Gamma}_{i}\right)\right) .
$$

Hence, the result follows from Equation (22) and Equation (23).

Lemma 4.10. Let $\Gamma$ be a metrized graph with an edge $e_{i} \in E(\Gamma)$ such that $\Gamma-e_{i}$ is connected. Let $p_{i}$ and $q_{i}$ be the end points of $e_{i}$. Then we have

$$
x(\Gamma)-y(\Gamma)=x\left(\bar{\Gamma}_{i}\right)-y\left(\bar{\Gamma}_{i}\right)+6 \frac{L_{i} A_{p_{i}, q_{i}, \Gamma-e_{i}}}{R_{i}\left(L_{i}+R_{i}\right)} .
$$

Proof. It follows from Lemma 3.1 and Lemma 3.4 that

$$
\tau\left(\bar{\Gamma}_{i}\right)=\tau\left(\Gamma-e_{i}\right)-\frac{R_{i}}{6}+\frac{A_{p_{i}, q_{i}, \Gamma-e_{i}}}{R_{i}} .
$$

From Equation (24) and Equation (20) applied to both $\bar{\Gamma}_{i}$ and $\Gamma-e_{i}$, we get

$$
x\left(\bar{\Gamma}_{i}\right)-y\left(\bar{\Gamma}_{i}\right)=x\left(\Gamma-e_{i}\right)-y\left(\Gamma-e_{i}\right)+R_{i}-6 \frac{A_{p_{i}, q_{i}, \Gamma-e_{i}}}{R_{i}} .
$$

Therefore, we obtain the result by solving Equation (25) for $x\left(\Gamma-e_{i}\right)-y\left(\Gamma-e_{i}\right)$ and substituting into Equation (23).

For a given metrized graph $\Gamma$ with $\#(E(\Gamma))=e$, let $\left\{L_{1}, L_{2}, \ldots, L_{e}\right\}$ be the edge lengths. Both of the functions $x: \mathbb{R}_{>0}^{e} \rightarrow \mathbb{R}$ given by $x\left(L_{1}, L_{2}, \ldots, L_{e}\right)=x(\Gamma)$ and $y$ : $\mathbb{R}_{>0}^{e} \rightarrow \mathbb{R}$ given by $y\left(L_{1}, L_{2}, \ldots, L_{e}\right)=y(\Gamma)$ are continuously differentiable homogeneous functions of degree 1 , when we consider all possible length distributions without changing the topology of $\Gamma$. 
Theorem 4.11. Let $\Gamma$ be a bridgeless metrized graph. Then we have

$$
\begin{aligned}
& x(\Gamma)=\sum_{e_{i} \in E(\Gamma)} \frac{L_{i} R_{i}^{2}}{\left(L_{i}+R_{i}\right)^{2}}+\sum_{e_{i} \in E(\Gamma)} \frac{L_{i} R_{i}}{\left(L_{i}+R_{i}\right)^{2}}\left(x\left(\Gamma-e_{i}\right)-x\left(\bar{\Gamma}_{i}\right)\right), \\
& y(\Gamma)=\sum_{e_{i} \in E(\Gamma)} \frac{L_{i} R_{i}}{\left(L_{i}+R_{i}\right)^{2}}\left(y\left(\Gamma-e_{i}\right)-y\left(\bar{\Gamma}_{i}\right)\right) .
\end{aligned}
$$

Proof. By taking the partial derivatives of the both sides of the equalities in Theorem 4.9 with respect to $L_{i}$ gives

$$
\begin{aligned}
& \frac{\partial x(\Gamma)}{\partial L_{i}}=\frac{R_{i}^{2}}{\left(L_{i}+R_{i}\right)^{2}}+\frac{R_{i}}{\left(L_{i}+R_{i}\right)^{2}} x\left(\Gamma-e_{i}\right)-\frac{R_{i}}{\left(L_{i}+R_{i}\right)^{2}} x\left(\bar{\Gamma}_{i}\right), \\
& \frac{\partial y(\Gamma)}{\partial L_{i}}=\frac{R_{i}}{\left(L_{i}+R_{i}\right)^{2}} y\left(\Gamma-e_{i}\right)-\frac{R_{i}}{\left(L_{i}+R_{i}\right)^{2}} y\left(\bar{\Gamma}_{i}\right) .
\end{aligned}
$$

Therefore, by applying Euler's formula we obtain the equalities we wanted.

We call the following identities the contraction identities for $x(\Gamma)$ and $y(\Gamma)$.

Theorem 4.12. Let $\Gamma$ be a bridgeless metrized graph with $v=\#(V(\Gamma)) \geqslant 2$. Then we have

$$
(v-2) x(\Gamma)=\sum_{e_{i} \in E(\Gamma)} \frac{R_{i}}{L_{i}+R_{i}} x\left(\bar{\Gamma}_{i}\right), \quad(v-2) y(\Gamma)=\sum_{e_{i} \in E(\Gamma)} \frac{R_{i}}{L_{i}+R_{i}} y\left(\bar{\Gamma}_{i}\right),
$$

Proof. Multiplying both sides of the equalities in Theorem 4.9 by $\frac{R_{i}}{L_{i}+R_{i}}$, and using the fact that $\sum_{e_{i} \in E(\Gamma)} \frac{R_{i}}{L_{i}+R_{i}}=v-1$ (see Equation (3)) we obtain

$$
\begin{aligned}
& (v-1) x(\Gamma)=\sum_{e_{i} \in E(\Gamma)} \frac{L_{i} R_{i}^{2}}{\left(L_{i}+R_{i}\right)^{2}}+\sum_{e_{i} \in E(\Gamma)} \frac{L_{i} R_{i}}{\left(L_{i}+R_{i}\right)^{2}} x\left(\Gamma-e_{i}\right)+\sum_{e_{i} \in E(\Gamma)} \frac{R_{i}^{2}}{\left(L_{i}+R_{i}\right)^{2}} x\left(\bar{\Gamma}_{i}\right), \\
& (v-1) y(\Gamma)=\sum_{e_{i} \in E(\Gamma)} \frac{L_{i} R_{i}}{\left(L_{i}+R_{i}\right)^{2}} y\left(\Gamma-e_{i}\right)+\sum_{e_{i} \in E(\Gamma)} \frac{R_{i}^{2}}{\left(L_{i}+R_{i}\right)^{2}} y\left(\bar{\Gamma}_{i}\right) .
\end{aligned}
$$

Thus, the result follows from Equation (27) and Theorem 4.11.

We call the first identity in the corollary below the contraction identity for $z(\Gamma)$.

Corollary 4.13. Let $\Gamma$ be a bridgeless metrized graph with $v=\#(V(\Gamma)) \geqslant 2$. Then we have

$$
(v-1) z(\Gamma)=\sum_{e_{i} \in E(\Gamma)} \frac{R_{i}}{L_{i}+R_{i}} z\left(\bar{\Gamma}_{i}\right), \quad(v-2) r(\Gamma)=\sum_{e_{i} \in E(\Gamma)} \frac{R_{i}}{L_{i}+R_{i}} r\left(\bar{\Gamma}_{i}\right) .
$$


Proof. The second equality follows by adding the expressions in Theorem 4.12 and using Equation (21). Using the second equality along with the facts that $z(\Gamma)=\ell(\Gamma)-r(\Gamma)$, $z\left(\bar{\Gamma}_{i}\right)=\ell(\Gamma)-L_{i}-r\left(\bar{\Gamma}_{i}\right)$ and $\sum_{e_{i} \in E(\Gamma)} \frac{R_{i}}{L_{i}+R_{i}}=v-1$ (see Equation (3)), we obtain the

first equality.

Corollary 4.14. Let $\Gamma$ be a bridgeless metrized graph with $v=\#(V(\Gamma)) \geqslant 3$. Then we have

$$
\tau(\Gamma)=\frac{\ell(\Gamma)}{12}-\frac{1}{6(v-2)} \sum_{e_{i} \in E(\Gamma)} \frac{R_{i}}{L_{i}+R_{i}}\left(x\left(\bar{\Gamma}_{i}\right)-y\left(\bar{\Gamma}_{i}\right)\right)
$$

Proof. By Theorem 4.12, we have

$$
(v-2)(x(\Gamma)-y(\Gamma))=\sum_{e_{i} \in E(\Gamma)} \frac{R_{i}}{L_{i}+R_{i}}\left(x\left(\bar{\Gamma}_{i}\right)-y\left(\bar{\Gamma}_{i}\right)\right) .
$$

Thus, the result follows from Equation (20).

We call the identities in Theorem 4.15 and Corollary 4.16 the deletion identities.

Theorem 4.15. Let $\Gamma$ be a bridgeless metrized graph. Then we have

$$
g \cdot x(\Gamma)=y(\Gamma)+\sum_{e_{i} \in E(\Gamma)} \frac{L_{i}}{L_{i}+R_{i}} x\left(\Gamma-e_{i}\right), \quad(g+1) y(\Gamma)=\sum_{e_{i} \in E(\Gamma)} \frac{L_{i}}{L_{i}+R_{i}} y\left(\Gamma-e_{i}\right) .
$$

Proof. Multiplying both sides of the equalities in Theorem 4.9 by $\frac{L_{i}}{L_{i}+R_{i}}$, and using the fact that $\sum_{e_{i} \in E(\Gamma)} \frac{L_{i}}{L_{i}+R_{i}}=g$ (see Equation (3)) we obtain

$$
\begin{aligned}
& g \cdot x(\Gamma)=\sum_{e_{i} \in E(\Gamma)} \frac{L_{i}^{2} R_{i}}{\left(L_{i}+R_{i}\right)^{2}}+\sum_{e_{i} \in E(\Gamma)} \frac{L_{i}^{2}}{\left(L_{i}+R_{i}\right)^{2}} x\left(\Gamma-e_{i}\right)+\sum_{e_{i} \in E(\Gamma)} \frac{L_{i} R_{i}}{\left(L_{i}+R_{i}\right)^{2}} x\left(\bar{\Gamma}_{i}\right), \\
& g \cdot y(\Gamma)=\sum_{e_{i} \in E(\Gamma)} \frac{L_{i}^{2}}{\left(L_{i}+R_{i}\right)^{2}} y\left(\Gamma-e_{i}\right)+\sum_{e_{i} \in E(\Gamma)} \frac{L_{i} R_{i}}{\left(L_{i}+R_{i}\right)^{2}} y\left(\bar{\Gamma}_{i}\right) .
\end{aligned}
$$

The first equality is obtained by adding the first equalities in Theorem 4.11 and Equation (29) and using the fact that $r(\Gamma)=x(\Gamma)+y(\Gamma)$.

Similarly, the second equality is obtained by adding the second equalities in Theorem 4.11 and Equation (29).

Corollary 4.16. Let $\Gamma$ be a bridgeless metrized graph. Then we have

$$
(g-1) z(\Gamma)=\sum_{e_{i} \in E(\Gamma)} \frac{L_{i}}{L_{i}+R_{i}} z\left(\Gamma-e_{i}\right), \quad g \cdot r(\Gamma)=\sum_{e_{i} \in E(\Gamma)} \frac{L_{i}}{L_{i}+R_{i}} r\left(\Gamma-e_{i}\right) .
$$


Proof. Adding the identities in Theorem 4.15 and using the fact that $r(\Gamma)=x(\Gamma)+y(\Gamma)$ give the second formula.

Then the first formula is obtained by using the second formula, Equation (3) and the fact that $\ell(\Gamma)=z(\Gamma)+r(\Gamma)$.

Corollary 4.17. Let $\Gamma$ be a bridgeless metrized graph. Then we have

$$
\begin{gathered}
\tau(\Gamma)=\frac{\ell(\Gamma)}{12}-\frac{1}{6(g+1)} \sum_{e_{i} \in E(\Gamma)} \frac{L_{i}}{L_{i}+R_{i}}\left(x\left(\Gamma-e_{i}\right)-y\left(\Gamma-e_{i}\right)\right) \\
-\frac{1}{6(g+1) g} \sum_{e_{i} \in E(\Gamma)} \frac{L_{i}}{L_{i}+R_{i}} r\left(\Gamma-e_{i}\right) .
\end{gathered}
$$

Proof. By Theorem 4.15 and the fact that $r(\Gamma)=x(\Gamma)+y(\Gamma)$, we have

$$
(g+1) \cdot(x(\Gamma)-y(\Gamma))=r(\Gamma)+\sum_{e_{i} \in E(\Gamma)} \frac{L_{i}}{L_{i}+R_{i}}\left(x\left(\Gamma-e_{i}\right)-y\left(\Gamma-e_{i}\right)\right) .
$$

Thus, the result follows from Equation (20) and the second identity in Corollary 4.16.

In this section, we proved the following identities among other things:

By Theorem 4.9 and Theorem 4.5, the contraction-deletion identities for a metrized graph $\Gamma$ and for an edge $e_{i} \in E(\Gamma)$ with connected $\Gamma-e_{i}$ are

$$
\begin{aligned}
& x(\Gamma)=\frac{L_{i} R_{i}}{L_{i}+R_{i}}+\frac{L_{i}}{L_{i}+R_{i}} x\left(\Gamma-e_{i}\right)+\frac{R_{i}}{L_{i}+R_{i}} x\left(\bar{\Gamma}_{i}\right), \\
& y(\Gamma)=\frac{L_{i}}{L_{i}+R_{i}} y\left(\Gamma-e_{i}\right)+\frac{R_{i}}{L_{i}+R_{i}} y\left(\bar{\Gamma}_{i}\right), \\
& z(\Gamma)=\frac{L_{i}^{2}}{L_{i}+R_{i}}+\frac{L_{i}}{L_{i}+R_{i}} z\left(\Gamma-e_{i}\right)+\frac{R_{i}}{L_{i}+R_{i}} z\left(\bar{\Gamma}_{i}\right) .
\end{aligned}
$$

By Theorem 4.12 and Corollary 4.13, the contraction identities for a bridgeless metrized graph with $v=\#(V(\Gamma)) \geqslant 2$ are

$$
\begin{array}{rlrl}
(v-2) x(\Gamma) & =\sum_{e_{i} \in E(\Gamma)} \frac{R_{i}}{L_{i}+R_{i}} x\left(\bar{\Gamma}_{i}\right), & & (v-2) y(\Gamma)=\sum_{e_{i} \in E(\Gamma)} \frac{R_{i}}{L_{i}+R_{i}} y\left(\bar{\Gamma}_{i}\right), \\
(v-1) z(\Gamma)=\sum_{e_{i} \in E(\Gamma)} \frac{R_{i}}{L_{i}+R_{i}} z\left(\bar{\Gamma}_{i}\right), & (v-2) r(\Gamma)=\sum_{e_{i} \in E(\Gamma)} \frac{R_{i}}{L_{i}+R_{i}} r\left(\bar{\Gamma}_{i}\right) .
\end{array}
$$

By Theorem 4.15 and Corollary 4.16, the deletion identities for a bridgeless $\Gamma$ are

$$
\begin{array}{ll}
g \cdot x(\Gamma)=y(\Gamma)+\sum_{e_{i} \in E(\Gamma)} \frac{L_{i}}{L_{i}+R_{i}} x\left(\Gamma-e_{i}\right), & (g+1) y(\Gamma)=\sum_{e_{i} \in E(\Gamma)} \frac{L_{i}}{L_{i}+R_{i}} y\left(\Gamma-e_{i}\right), \\
(g-1) z(\Gamma)=\sum_{e_{i} \in E(\Gamma)} \frac{L_{i}}{L_{i}+R_{i}} z\left(\Gamma-e_{i}\right), & g \cdot r(\Gamma)=\sum_{e_{i} \in E(\Gamma)} \frac{L_{i}}{L_{i}+R_{i}} r\left(\Gamma-e_{i}\right) .
\end{array}
$$


Also, for a bridgeless $\Gamma$ the following identity of Theorem 4.7 deserves attention:

$$
\sum_{e_{i} \in E(\Gamma)} \frac{L_{i} K_{i}(\Gamma)}{L_{i}+R_{i}}=\sum_{e_{i} \in E(\Gamma)} \frac{L_{i} R_{i}}{\left(L_{i}+R_{i}\right)^{2}}\left(z\left(\bar{\Gamma}_{i}\right)-z\left(\Gamma-e_{i}\right)\right)=\sum_{e_{i} \in E(\Gamma)} \frac{L_{i}^{2} R_{i}}{\left(L_{i}+R_{i}\right)^{2}} .
$$

\section{Successive edge contraction}

In this section, we will successively contract edges in $E(\Gamma)$ for any metrized graph $\Gamma$. The contraction identities developed in the previous section will enable us to generalize the results of $\S 3$ and some of the results of $\S 4$. The results of this section will help us to understand the effects of topological properties of $\Gamma$, such as the edge connectivity, on $\tau(\Gamma)$.

Let $\Gamma$ be a metrized graph and let $\bar{\Gamma}_{i}$ be the metrized graph obtained by contracting $i$-th edge $e_{i} \in E(\Gamma)$ to its end points. Similarly, for any integer $k \geqslant 2$, let $\bar{\Gamma}_{i_{1}, i_{2}, \ldots, i_{k}}$ be the metrized graph obtained by contracting $i_{k}$-th edge $e_{i_{k}} \in E\left(\bar{\Gamma}_{i_{1}, i_{2}, \ldots, i_{k-1}}\right)$ to its end points. Note that $E\left(\bar{\Gamma}_{i_{1}, i_{2}, \ldots, i_{k}}\right)=E(\Gamma)-\left\{e_{i_{1}}, e_{i_{2}}, \ldots, e_{i_{k}}\right\}$ for any $k$. Let $\bar{\Gamma}_{i_{0}}:=\Gamma$.

Let $e_{i_{k}} \in E(\Gamma)$ be an edge of index $i_{k}$. Recall that we denote the resistance between the end points of $e_{i_{k}}$ in $\Gamma-e_{i_{k}}$ by $R_{i_{k}}$ and that we use $L_{i_{k}}$ to denote the length of $e_{i_{k}}$.

Now, we generalize Equation (28) as follows:

Lemma 5.1. Let $\Gamma$ be a bridgeless metrized graph with $(k+2) \leqslant v=\#(V(\Gamma))$ for some integer $k \geqslant 1$. Then

$$
\begin{gathered}
\frac{(v-2) !}{(v-k-2) !}(x(\Gamma)-y(\Gamma))=\sum_{e_{i_{1}} \in E(\Gamma)} \frac{R_{i_{1}}}{L_{i_{1}}+R_{i_{1}}} \sum_{\substack{e_{i_{2}} \in \\
E\left(\bar{\Gamma}_{i_{1}}\right)}} \frac{R_{i_{2}}}{L_{i_{2}}+R_{i_{2}}} \ldots \\
\sum_{e_{i_{k}} \in E\left(\bar{\Gamma}_{\left.i_{1}, \ldots, i_{k-1}\right)}\right.} \frac{R_{i_{k}}}{L_{i_{k}}+R_{i_{k}}}\left(x\left(\bar{\Gamma}_{i_{1}, \ldots, i_{k}}\right)-y\left(\bar{\Gamma}_{i_{1}, \ldots, i_{k}}\right)\right) .
\end{gathered}
$$

Proof. Note that if an edge of a bridgeless graph is contracted the resulting graph will be also bridgeless. Contraction of a self loop does not change the number of vertices. However, if an edge $e_{i_{j}}$ is a self loop, then $\frac{R_{i_{j}}}{L_{i_{j}}+R_{i_{j}}}=0$. Thus, contraction of self loops does not contribute to sums in contraction identities. Hence, we can inductively apply Equation (28) to obtain the result.

Multiple edges or self loops may appear after contracting an edge, but this does not cause any problem for any of the contraction identities.

We can generalize Corollary 4.14 as follows:

Theorem 5.2. Let $\Gamma$ be a bridgeless metrized graph with $(k+2) \leqslant v=\#(V(\Gamma))$ for some integer $k \geqslant 1$. Then we have 


$$
\begin{gathered}
\tau(\Gamma)=\frac{\ell(\Gamma)}{12}-\frac{(v-k-2) !}{6(v-2) !} \sum_{e_{i_{1}} \in E(\Gamma)} \frac{R_{i_{1}}}{L_{i_{1}}+R_{i_{1}}} \sum_{\substack{e_{i_{2}} \in \\
E\left(\bar{\Gamma}_{i_{1}}\right)}} \frac{R_{i_{2}}}{L_{i_{2}}+R_{i_{2}}} \ldots \\
\sum_{e_{i_{k}} \in E\left(\bar{\Gamma}_{i_{1}, \ldots, i_{k-1}}\right)} \frac{R_{i_{k}}}{L_{i_{k}}+R_{i_{k}}}\left(x\left(\bar{\Gamma}_{i_{1}, \ldots, i_{k}}\right)-y\left(\bar{\Gamma}_{i_{1}, \ldots, i_{k}}\right)\right) .
\end{gathered}
$$

Proof. We can use Lemma 5.1 and Equation (20) to obtain the result.

Here is another formula for $r(\Gamma)$ :

Proposition 5.3. Let $\Gamma$ be a bridgeless graph with $3 \leqslant v=\#(V(\Gamma))$. Then for any $k$ with $k+2 \leqslant v$,

$$
\frac{k(v-2) !}{(v-k-1) !} r(\Gamma)=\sum_{e_{i_{1}} \in E(\Gamma)} \frac{R_{i_{1}}}{L_{i_{1}}+R_{i_{1}}} \cdots \sum_{\substack{e_{i_{k}} \in \\ E\left(\bar{\Gamma}_{i_{1}, \ldots, i_{k-1}}\right)}} \frac{R_{i_{k}}}{L_{i_{k}}+R_{i_{k}}} \sum_{t=1}^{k} L_{i_{t}} .
$$

Proof. By applying the second part of Corollary 4.13 successively, we obtain

$$
\frac{(v-2) !}{(v-k-2) !} r(\Gamma)=\sum_{e_{i_{1}} \in E(\Gamma)} \frac{R_{i_{1}}}{L_{i_{1}}+R_{i_{1}}} \cdots \sum_{\substack{e_{i_{k}} \in \\ E\left(\bar{\Gamma}_{i_{1}, \ldots, i_{k-1}}\right)}} \frac{R_{i_{k}}}{L_{i_{k}}+R_{i_{k}}} r\left(\bar{\Gamma}_{i_{1}, \ldots, i_{k}}\right) .
$$

Now, we can use induction on $k$ to show the identity in the proposition. When $k=1$, the result holds trivially by the definition of $r(\Gamma)$. Suppose the result is true for $k=n$ where $n+3 \leqslant v$. Let $A$ be the right hand side of Equation (35) for $k=n+1$. By splitting the $\operatorname{sum} \sum_{t=1}^{n+1} L_{i_{t}}=\left(\sum_{t=1}^{n} L_{i_{t}}\right)+L_{i_{n+1}}$ we have

$$
\begin{aligned}
A= & \sum_{\substack{\left.e_{i_{1}} \in\right) \\
E(\Gamma)}} \frac{R_{i_{1}}}{L_{i_{1}}+R_{i_{1}}} \cdots \sum_{\substack{e_{i_{n}} \in \\
E\left(\bar{\Gamma}_{i_{1}, \ldots, i_{n-1}}\right)}} \frac{R_{i_{n}}}{L_{i_{n}}+R_{i_{n}}} \sum_{t=1}^{n} L_{i_{t}} \sum_{\substack{e_{i_{n+1}} \in \\
E\left(\overline{\Gamma_{i_{1}}, \ldots, i_{n}}\right.}} \frac{R_{i_{n+1}}}{L_{i_{n+1}}+R_{i_{n+1}}} \\
& +\sum_{\substack{e_{i_{1}} \in \\
E(\Gamma)}} \frac{R_{i_{1}}}{L_{i_{1}}+R_{i_{1}}} \cdots \sum_{\substack{e_{i_{n}} \in \\
E\left(\bar{\Gamma}_{i_{1}, \ldots, i_{n-1}}\right)}} \frac{R_{i_{n}}}{L_{i_{n}}+R_{i_{n}}} r\left(\bar{\Gamma}_{i_{1}, \ldots, i_{n}}\right) \\
= & (v-n-1) \sum_{\substack{e_{i_{1}} \in \\
E(\Gamma)}} \frac{R_{i_{1}}}{L_{i_{1}}+R_{i_{1}}} \cdots \sum_{E\left(\bar{\Gamma}_{i_{1}, \ldots, i_{n-1}}\right)} \frac{R_{i_{n}}}{L_{i_{n}}+R_{i_{n}}} \sum_{t=1}^{n} L_{i_{t}}+\frac{(v-2) !}{(v-n-2) !} r(\Gamma)
\end{aligned}
$$

by Equation (3) applied to $\bar{\Gamma}_{i_{1}, \ldots, i_{n}}$, and by Equation (36).

$$
=\frac{(n+1)(v-2) !}{(v-n-2) !} r(\Gamma), \quad \text { by the induction assumption. }
$$

Hence the result follows. 
Note that Equation (36) generalizes the second equation in Corollary 4.13.

Corollary 5.4. Let $\Gamma$ be a bridgeless graph with $3 \leqslant v=\#(V(\Gamma))$. Then

$$
(v-2)(v-2) ! r(\Gamma)=\sum_{\substack{e_{i_{1}} \in \\ E(\Gamma)}} \frac{R_{i_{1}}}{L_{i_{1}}+R_{i_{1}}} \cdots \sum_{\substack{e_{i_{v-2}} \in \\ E\left(\bar{\Gamma}_{i_{1}, \ldots, i_{v-3}}\right)}} \frac{R_{i_{v-2}}}{L_{i_{v-2}}+R_{i_{v-2}}} \sum_{t=1}^{v-2} L_{i_{t}} .
$$

Proof. The result follows from Proposition 5.3 with $k=v-2$.

Corollary 5.5. Let $\Gamma$ be a bridgeless graph with $3 \leqslant v=\#(V(\Gamma))$ and e edges. For any $k \in\{1,2, \ldots, v-2\}$, let $A_{k}=\left\{\sum_{t=1}^{k} L_{i_{t}} \mid\left\{i_{1}, \ldots, i_{k}\right\} \subseteq\{1,2, \ldots, e\}\right\}$. Let $C_{k}=\max \left(A_{k}\right)$ and $c_{k}=\min \left(A_{k}\right)$. Then we have

$$
\frac{(v-1)}{k} c_{k} \leqslant r(\Gamma) \leqslant \frac{(v-1)}{k} C_{k}, \text { and in particular, } \quad \frac{v-1}{v-2} c_{v-2} \leqslant r(\Gamma) \leqslant \frac{v-1}{v-2} C_{v-2} .
$$

Proof. The result follows from Proposition 5.3 and Equation (3).

Note also that successive application of the first part of Corollary 4.13 gives

$$
\frac{(v-1) !}{(v-k-1) !} z(\Gamma)=\sum_{\substack{e_{i_{1}} \in \\ E(\Gamma)}} \frac{R_{i_{1}}}{L_{i_{1}}+R_{i_{1}}} \cdots \sum_{\substack{e_{i_{k}} \in \\ E\left(\bar{\Gamma}_{i_{1}, \ldots, i_{k-1}}\right)}} \frac{R_{i_{k}}}{L_{i_{k}}+R_{i_{k}}} z\left(\bar{\Gamma}_{i_{1}, \ldots, i_{k}}\right) .
$$

The following theorem generalizes Theorem 3.3.

Theorem 5.6. Let $\Gamma$ be a bridgeless metrized graph with $(k+2) \leqslant v=\#(V(\Gamma))$ for some integer $k \geqslant 1$. Then

$\tau(\Gamma)=\frac{(v-k-2) !}{(v-2) !} \sum_{e_{i_{1}} \in E(\Gamma)} \frac{R_{i_{1}}}{L_{i_{1}}+R_{i_{1}}} \cdots \sum_{\substack{e_{i_{k}} \in \\ E\left(\bar{\Gamma}_{i_{1}, \ldots, i_{k-1}}\right)}} \frac{R_{i_{k}}}{L_{i_{k}}+R_{i_{k}}} \tau\left(\bar{\Gamma}_{i_{1}, \ldots, i_{k}}\right)-\frac{k \cdot z(\Gamma)}{12(v-k-1)}$

Proof. First, we note that $\tau\left(\bar{\Gamma}_{i_{1}, \ldots, i_{k}}\right)=\frac{\ell\left(\bar{\Gamma}_{i_{1}, \ldots, i_{k}}\right)}{12}-\frac{x\left(\bar{\Gamma}_{i_{1}, \ldots, i_{k}}\right)-y\left(\bar{\Gamma}_{i_{1}, \ldots, i_{k}}\right)}{6}$ by Equation (20), and $\ell\left(\bar{\Gamma}_{i_{1}, \ldots, i_{k}}\right)=\ell(\Gamma)-\sum_{t=1}^{k} L_{i_{t}}$. Then the result follows by applying Theorem 5.2 and Proposition 5.3.

Corollary 5.7. Let $\Gamma$ be a bridgeless metrized graph with $3 \leqslant v=\#(V(\Gamma))$. Then

$$
\tau(\Gamma)=\frac{1}{(v-2) !} \sum_{\substack{e_{i_{1}} \in \\ E(\Gamma)}} \frac{R_{i_{1}}}{L_{i_{1}}+R_{i_{1}}} \cdots \sum_{\substack{e_{i_{v-2}} \in \\ E\left(\bar{\Gamma}_{i_{1}, \ldots, i_{v-3}}\right)}} \frac{R_{i_{v-2}}}{L_{i_{v-2}}+R_{i_{v-2}}} \tau\left(\bar{\Gamma}_{i_{1}, \ldots, i_{v-2}}\right)-\frac{v-2}{12} z(\Gamma) .
$$

Proof. The result follows from Theorem 5.6 with $k=v-2$. 


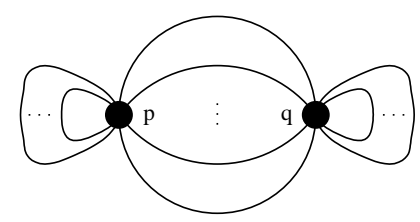

Figure 3: A Banana graph with self loops.

Recall that Theorem 3.3 is valid for graphs with more than 2 vertices. If an edge $e_{i_{k}}$ is not a self loop in $\bar{\Gamma}_{i_{1}, i_{2}, \ldots, i_{k-1}}$, then $\#\left(V\left(\bar{\Gamma}_{i_{1}, i_{2}, \ldots, i_{k}}\right)\right)=\#\left(V\left(\bar{\Gamma}_{i_{1}, i_{2}, \ldots, i_{k-1}}\right)\right)-1$. We call $\bar{\Gamma}_{i_{1}, i_{2}, \ldots, i_{v-2}}$ an admissible contraction of $\Gamma$, if it is obtained from $\Gamma$ by contracting edges with distinct end points, i.e., if we have $\#\left(V\left(\bar{\Gamma}_{i_{1}, i_{2}, \ldots, i_{v-2}}\right)\right)=2$. Note that such graphs are the only ones that contribute to the sum in Corollary 5.7.

Let $\bar{\Gamma}_{i_{1}, i_{2}, \ldots, i_{v-2}}$ be an admissible contraction of $\Gamma$, and let $V\left(\bar{\Gamma}_{i_{1}, \ldots, i_{v-2}}\right)=\{p, q\}$. Let $n^{\prime}:=n\left(i_{1}, \ldots, i_{v-2}\right)$ be the number of multiple edges in $\bar{\Gamma}_{i_{1}, \ldots, i_{v-2}}$, and let $B^{\prime}:=$ $\left\{e_{j_{1}}, e_{j_{2}}, \ldots e_{j_{n^{\prime}}}\right\}$ be the set of multiple edges in $\bar{\Gamma}_{i_{1}, \ldots, i_{v-2}}$. Note that $\bar{\Gamma}_{i_{1}, \ldots, i_{v-2}}$ possibly has self-loops at $p$ or $q$. Figure 3 illustrates $\bar{\Gamma}_{i_{1}, \ldots, i_{v-2}}$. For the resistance function $r^{\prime}(x, y)$ in $\bar{\Gamma}_{i_{1}, \ldots, i_{v-2}}$, we have $r^{\prime}(p, q)=\frac{1}{\sum_{t=1}^{n^{\prime}} \frac{1}{L_{j_{t}}}}$ by circuit theory. Therefore,

Proposition 5.8. Let $\Gamma$ be a bridgeless metrized graph. Using the notation above, for each admissible contraction $\bar{\Gamma}_{i_{1}, \ldots, i_{v-2}}$ of $\Gamma$, we have

$$
x\left(\bar{\Gamma}_{i_{1}, \ldots, i_{v-2}}\right)=\left(n^{\prime}-1\right) \cdot r^{\prime}(p, q), \quad y\left(\bar{\Gamma}_{i_{1}, \ldots, i_{v-2}}\right)=r^{\prime}(p, q) .
$$

Proof. First note that $r^{\prime}(p, q)=\frac{L_{t} R_{t}}{L_{t}+R_{t}}$ for each $e_{t} \in B^{\prime}$, and $R_{i_{t}}=0$ if $e_{i_{t}} \notin B^{\prime}$.

$$
r\left(\bar{\Gamma}_{i_{1}, \ldots, i_{v-2}}\right)=\sum_{e_{t} \in B^{\prime}} \frac{L_{t} R_{t}}{L_{t}+R_{t}}=n^{\prime} \cdot r^{\prime}(p, q) .
$$

Moreover, $\left(R_{a_{i}, p}-R_{b_{i}, p}\right)^{2}=R_{i}^{2}$ for each $e_{i} \in E\left(\bar{\Gamma}_{i_{1}, \ldots, i_{v-2}}\right)$. Thus, by definition

$$
\begin{aligned}
x\left(\bar{\Gamma}_{i_{1}, \ldots, i_{v-2}}\right) & =\sum_{e_{i_{v-1}} \in E\left(\bar{\Gamma}_{i_{1}, \ldots, i_{v-2}}\right)} \frac{L_{i_{v-1}}^{2} R_{i_{v-1}}}{\left(L_{i_{v-1}}+R_{i_{v-1}}\right)^{2}}=\sum_{e_{t} \in B^{\prime}} \frac{L_{t}^{2} R_{t}}{\left(L_{t}+R_{t}\right)^{2}} \\
& =r^{\prime}(p, q) \sum_{e_{t} \in B^{\prime}} \frac{L_{t}}{L_{t}+R_{t}}=r^{\prime}(p, q)\left(n^{\prime}-1\right),
\end{aligned}
$$

where the last equality follows from Equation (3). This proves the first equality, and the second equality follows from the first equality and Equation (38).

The following theorem gives another formula for the tau constant, and it is in some sense an improved version of Theorem 2.6. 
Theorem 5.9. Let $\Gamma$ be a bridgeless metrized graph with $3 \leqslant v=\#(E(\Gamma))$. Let $p, q, n^{\prime}$ and $B^{\prime}$ be as defined above.

$$
\tau(\Gamma)=\frac{\ell(\Gamma)}{12}-\frac{1}{6 \cdot(v-2) !} \sum_{e_{i_{1}} \in E(\Gamma)} \frac{R_{i_{1}}}{L_{i_{1}}+R_{i_{1}}} \cdots \sum_{\substack{e_{i_{v-2}} \in \\ E\left(\bar{\Gamma}_{i_{1}, \ldots, i_{v-3}}\right)}} \frac{R_{i_{v-2}}}{L_{i_{v-2}}+R_{i_{v-2}}}\left(n^{\prime}-2\right) r^{\prime}(p, q) .
$$

Proof. We have $x\left(\bar{\Gamma}_{i_{1}, \ldots, i_{v-2}}\right)-y\left(\bar{\Gamma}_{i_{1}, \ldots, i_{v-2}}\right)=\left(n^{\prime}-2\right) \cdot r^{\prime}(p, q)$, by Proposition 5.8 . Therefore, we obtain what we want by using Theorem 5.2 with $k=v-2$.

\section{Edge connectivity and the tau constant}

In this section, we will prove that Conjecture 1.1 holds with $C=\frac{1}{108}$ for any graph $\Gamma$ with edge connectivity more than or equal to 6 , and we will give a lower bound to the tau constant in terms of edge connectivity.

Let $\Gamma$ be a bridgeless metrized graph, and let $\bar{\Gamma}_{i_{1}, \ldots, i_{v-2}}, n^{\prime}, p, q, r^{\prime}(p, q)$ and $B^{\prime}$ be as in $\S 5$. Recall that $n^{\prime}:=n\left(i_{1}, \ldots, i_{v-2}\right)$ is the number of multiple edges in $\bar{\Gamma}_{i_{1}, \ldots, i_{v-2}}$ and that $B^{\prime}:=\left\{e_{1}, e_{2}, \ldots, e_{n^{\prime}}\right\}$ is the set of multiple edges in $\bar{\Gamma}_{i_{1}, \ldots, i_{v-2}}$. We will show that a lower bound for

$$
N(\Gamma):=\min \left\{n^{\prime} \mid\left\{i_{1}, \ldots, i_{v-2}\right\} \subset\{1,2, \ldots, e\}\right\} .
$$

gives a lower bound for $\tau(\Gamma)$. We will make some observations about $N(\Gamma)$ after recalling some basic definitions from graph theory.

We recall the following inequality between the edge connectivity $\Lambda(\Gamma)$, vertex connectivity $\kappa(\Gamma)$, and the minimum degree of the valences $\delta(\Gamma)$.

Lemma 6.1. [1, pg. 3] For a graph $\Gamma$, we have $\kappa(\Gamma) \leqslant \Lambda(\Gamma) \leqslant \delta(\Gamma)$.

Recall that a metrized graph is connected by definition.

Lemma 6.2. Let $\Gamma$ be a graph. Then $N(\Gamma)=\Lambda(\Gamma)$.

Proof. If $\# V(\Gamma)=2$, then $\Gamma$ is a banana graph with possibly self-loops. Then $N(\Gamma)=$ $\Lambda(\Gamma)$ clearly.

Note that when we contract an edge of a graph $\Gamma$ with $V(\Gamma) \geqslant 3$, the edge connectivity either does not change or increases. Therefore, $\Lambda\left(\bar{\Gamma}_{i_{1}, \ldots, i_{v-2}}\right) \geqslant \Lambda(\Gamma)$ for the contraction of any edges $e_{i_{1}}, \ldots, e_{i_{v-2}}$. Since $n^{\prime}=\Lambda\left(\bar{\Gamma}_{i_{1}, \ldots, i_{v-2}}\right) \geqslant \Lambda(\Gamma)$, we have $N(\Gamma) \geqslant \Lambda(\Gamma)$.

Let $k=\Lambda(\Gamma)$, and let $e_{1}, e_{2}, \ldots, e_{k}$ be edges such that $\Gamma-\left\{e_{1}, e_{2}, \ldots, e_{k}\right\}$ is disconnected but $\Gamma-\left(\left\{e_{1}, e_{2}, \ldots, e_{k}\right\}-e_{j}\right)$ is connected for each $e_{j}$ where $1 \leqslant j \leqslant k$. Also, let $p$ and $q$ be the end points of the edge $e_{k}$. Note that $e_{k}$ is a bridge in $\Gamma-\left\{e_{1}, e_{2}, \ldots, e_{k-1}\right\}$. That is, $\Gamma-\left\{e_{1}, e_{2}, \ldots, e_{k-1}\right\}=\beta \cup e_{k} \cup \gamma$ for some graphs $\beta$ and $\gamma$ with $\beta \cap e_{k}=\{p\}$ and $\gamma \cap e_{k}=\{q\}$. Let $\beta$ and $\gamma$ have $s+1$ and $t+1$ vertices, respectively. Contract edges in $E(\beta)$, say $e_{i_{1}}, e_{i_{2}}, \ldots, e_{i_{s}}$, one by one until $\beta$ has 1 vertex. This can be done by contracting only the edges that are not self loops at any step of this contraction process. Similarly, contract edges in $E(\gamma)$, say $e_{l_{1}}, e_{l_{2}}, \ldots, e_{l_{t}}$, one by one until $\gamma$ has 1 vertex. Then, 
$s+t=v-2$ and $n\left(i_{1}, i_{2}, \ldots, i_{s}, l_{1}, l_{2}, \ldots, l_{t}\right)=k$ for the contraction graph $\bar{\Gamma}_{i_{1}, i_{2}, \ldots, i_{s}, l_{1}, l_{2}, \ldots, l_{t}}$. That is, we obtain a banana graph with vertices $p$ and $q$, and bridges $e_{1}, e_{2}, \ldots, e_{k}$ after the contractions. Thus, $N(\Gamma) \leqslant \Lambda(\Gamma)$.

Hence, the result follows.

We will need the following computation before we relate the edge connectivity $\Lambda(\Gamma)$ to $\tau(\Gamma)$.

Corollary 6.3. Let $\Gamma$ be a bridgeless metrized graph with genus $g$. Then for any admissible contraction $\bar{\Gamma}_{i_{1}, \ldots, i_{v-2}}$ of $\Gamma$ we have

$$
g \cdot y\left(\bar{\Gamma}_{i_{1}, \ldots, i_{v-2}}\right) \geqslant x\left(\bar{\Gamma}_{i_{1}, \ldots, i_{v-2}}\right) \geqslant(\Lambda(\Gamma)-1) \cdot y\left(\bar{\Gamma}_{i_{1}, \ldots, i_{v-2}}\right) .
$$

Proof. Since $\bar{\Gamma}_{i_{1}, \ldots, i_{v-2}}$ has $e-(v-2)=g+1$ edges, $g+1 \geqslant \max \left\{n^{\prime} \mid\left\{i_{1}, \ldots, i_{v-2}\right\} \subset\right.$ $\{1,2, \ldots, e\}\}$. Then the first inequality follows from Proposition 5.8. The second inequality follows from Lemma 6.2 and Proposition 5.8.

When $k=v-2$, Equation (37) becomes

$$
(v-1) ! z(\Gamma)=\sum_{\substack{e_{i_{1}} \in \\ E(\Gamma)}} \frac{R_{i_{1}}}{L_{i_{1}}+R_{i_{1}}} \cdots \sum_{\substack{e_{i_{v-2}} \in \\ E\left(\bar{\Gamma}_{i_{1}, \ldots, i_{v-3}}\right)}} \frac{R_{i_{v-2}}}{L_{i_{v-2}}+R_{i_{v-2}}} \sum_{\substack{e_{i_{v-1}} \in \\ E\left(\bar{\Gamma}_{i_{1}, \ldots, i_{v-2}}\right)}} \frac{L_{i_{v-1}}^{2}}{L_{i_{v-1}}+R_{i_{v-1}}}
$$

Lemma 6.4. For each admissible contraction $\bar{\Gamma}_{i_{1}, \ldots, i_{v-2}}$ of $\Gamma$ as above we have

$$
\sum_{e_{t} \in B^{\prime}} \frac{L_{t}^{2}}{L_{t}+R_{t}} \geqslant n^{\prime} \cdot\left(n^{\prime}-1\right) r^{\prime}(p, q)
$$

Proof. We have $\frac{1}{n^{\prime}} \sum_{e_{t} \in B^{\prime}} L_{t} \geqslant \frac{n^{\prime}}{\sum_{e_{t} \in B^{\prime}} \frac{1}{L_{t}}}$ by Arithmetic-Harmonic Mean inequality. On the other hand, $\sum_{e_{t} \in B^{\prime}} L_{t}=\sum_{e_{t} \in B^{\prime}} \frac{L_{t}^{2}}{L_{t}+R_{t}}+\sum_{e_{t} \in B^{\prime}} \frac{L_{t} R_{t}}{L_{t}+R_{t}}$, and $r^{\prime}(p, q)=\frac{1}{\sum_{t=1}^{n^{\prime}} \frac{1}{L_{j_{t}}}}$. Thus the result follows from Equation (38).

Lemma 6.5. Let $\Gamma$ be a bridgeless metrized graph. Then we have $z(\Gamma) \geqslant \frac{\Lambda(\Gamma)}{v-1} x(\Gamma)$.

Proof. If we apply the first part of Theorem 4.12 successively, we derive the following expression:

$$
x(\Gamma)=\frac{1}{(v-2) !} \sum_{e_{i_{1}} \in E(\Gamma)} \frac{R_{i_{1}}}{L_{i_{1}}+R_{i_{1}}} \cdots \sum_{\substack{e_{i_{v-2}} \in \\ E\left(\bar{\Gamma}_{i_{1}, \ldots, i_{v-3}}\right)}} \frac{R_{i_{v-2}}}{L_{i_{v-2}}+R_{i_{v-2}}} x\left(\bar{\Gamma}_{i_{1}, \ldots, i_{v-2}}\right) .
$$

On the other hand, for each admissible contraction $\bar{\Gamma}_{i_{1}, \ldots, i_{v-2}}$ of $\Gamma, x\left(\bar{\Gamma}_{i_{1}, \ldots, i_{v-2}}\right)=\left(n^{\prime}-\right.$ 1) $r^{\prime}(p, q)$ by Proposition 5.8. Then the result follows from Equation (39), Lemma 6.2, Lemma 6.4, and Equation (40). 
Set

$$
w(\Gamma):=\frac{1}{(v-2) !} \sum_{\substack{e_{i_{1}} \in \\ E(\Gamma)}} \frac{R_{i_{1}}}{L_{i_{1}}+R_{i_{1}}} \cdots \sum_{\substack{e_{i_{v-2}} \in \\ E\left(\bar{\Gamma}_{i_{1}, \ldots, i_{v-3}}\right)}} \frac{R_{i_{v-2}}}{L_{i_{v-2}}+R_{i_{v-2}}} \sum_{\substack{e_{i_{v-1}} \in \\ E\left(\bar{\Gamma}_{i_{1}, \ldots, i_{v-2}}\right)}} \frac{L_{i_{v-1}}^{3}}{\left(L_{i_{v-1}}+R_{i_{v-1}}\right)^{2}} .
$$

Then we have

Lemma 6.6. Let $\Gamma$ be a bridgeless metrized graph. Then $(v-1) z(\Gamma)=w(\Gamma)+x(\Gamma)$.

Proof. The result follows from Equations (39) and (40), and using the fact that

$$
x\left(\bar{\Gamma}_{i_{1}, \ldots, i_{v-2}}\right)=\sum_{e_{i_{v-1}} \in E\left(\bar{\Gamma}_{i_{1}, \ldots, i_{v-2}}\right)} \frac{L_{i_{v-1}}^{2} R_{i_{v-1}}}{\left(L_{i_{v-1}}+R_{i_{v-1}}\right)^{2}}
$$

(cf. proof of Proposition 5.8).

Lemma 6.7. [5, Lemma 2.26] Let $\Gamma$ be a normalized metrized graph. Then

$$
\sum_{e_{i} \in E(\Gamma)} \frac{L_{i} R_{i}^{2}}{\left(L_{i}+R_{i}\right)^{2}} \geqslant\left(\sum_{e_{i} \in E(\Gamma)} \frac{L_{i} R_{i}}{L_{i}+R_{i}}\right)^{2}
$$

Lemma 6.8. [5, Lemma 2.12] Let $\Gamma$ be a metrized graph and $p \in V(\Gamma)$. Then if $e_{i} \sim p$ indicates that edge $e_{i}$ is incident to vertex $p$

$$
\sum_{e_{i} \in E(\Gamma)} \frac{L_{i}\left(R_{a_{i}, p}-R_{b_{i}, p}\right)^{2}}{\left(L_{i}+R_{i}\right)^{2}}=\frac{2}{v} \sum_{e_{i} \in E(\Gamma)} \frac{L_{i} R_{i}^{2}}{\left(L_{i}+R_{i}\right)^{2}}+\frac{1}{v} \sum_{p \in V(\Gamma)}\left(\sum_{\substack{e_{i} \not p \\ e_{i} \in E(\Gamma)}} \frac{L_{i}\left(R_{a_{i}, p}-R_{b_{i}, p}\right)^{2}}{\left(L_{i}+R_{i}\right)^{2}}\right) .
$$

We have the following relations between $x(\Gamma)$ and $y(\Gamma)$ :

Theorem 6.9. Let $\Gamma$ be a normalized bridgeless metrized graph with $\#(V(\Gamma))=v$, and let $x=x(\Gamma), y=y(\Gamma)$. Then we have

1. $\tau(\Gamma)=\frac{1}{12}-\frac{x}{6}+\frac{y}{6}$,

2. $1 \geqslant \frac{\Lambda(\Gamma)+v-1}{v-1} x+y, \quad x \geqslant 0$, and $y \geqslant 0$,

3. $y \geqslant \frac{v+6}{4 v}(x+y)^{2}$,

4. $g \cdot y \geqslant x \geqslant(\Lambda(\Gamma)-1) y$.

Proof. Since $\Gamma$ is normalized, $\ell(\Gamma)=1$. Thus, part (1) follows from Equation (20).

Part (2) follows from Lemma 6.5 and Equation (21).

By Lemma 6.8 and the definition of $y$, we have

$$
y \geqslant \frac{v+6}{4 v} \sum_{e_{i} \in E(\Gamma)} \frac{L_{i} R_{i}^{2}}{\left(L_{i}+R_{i}\right)^{2}}
$$




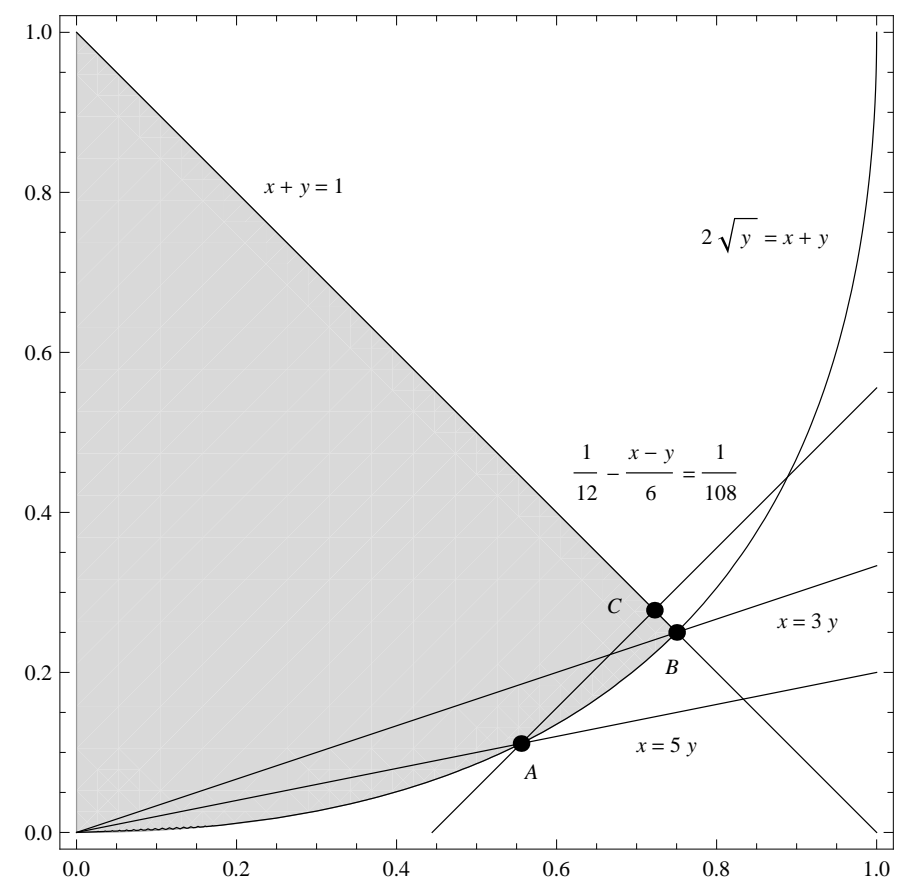

Figure 4: The lower bound for $\tau(\Gamma)$ when $\Lambda(\Gamma) \geqslant 6$ and $v \rightarrow \infty$. $A=\left(\frac{5}{9}, \frac{1}{9}\right), B=\left(\frac{3}{4}, \frac{1}{4}\right)$, $C=\left(\frac{13}{18}, \frac{5}{18}\right)$. Shaded part illustrates $x+y \leqslant 1$ and $2 \sqrt{y} \geqslant x+y$.

Thus, part (3) follows from Equation (41) and Lemma 6.7.

We have $g \cdot y\left(\bar{\Gamma}_{i_{1}, \ldots, i_{v-2}}\right) \geqslant x\left(\bar{\Gamma}_{i_{1}, \ldots, i_{v-2}}\right) \geqslant(\Lambda(\Gamma)-1) \cdot y\left(\bar{\Gamma}_{i_{1}, \ldots, i_{v-2}}\right)$ by Corollary 6.3. We inductively apply Theorem 4.12 to obtain

$$
(v-2) ! y(\Gamma)=\sum_{e_{i_{1}} \in E(\Gamma)} \frac{R_{i_{1}}}{L_{i_{1}}+R_{i_{1}}} \sum_{\substack{e_{i_{2}} \in \\ E\left(\bar{\Gamma}_{i_{1}}\right)}} \frac{R_{i_{2}}}{L_{i_{2}}+R_{i_{2}}} \cdots \sum_{\substack{e_{i_{v-2}} \in \\ E\left(\bar{\Gamma}_{i_{1}, \ldots, i_{v-3}}\right)}} \frac{R_{i_{i_{v}}}}{L_{i_{v-2}}+R_{i_{v-2}}} y\left(\bar{\Gamma}_{i_{1}, \ldots, i_{v-2}}\right) .
$$

Thus, using Equation (40) we have part (4).

Now, we can state the main result of this paper:

Theorem 6.10. Let $\Gamma$ be a metrized graph with $v$ vertices. Then we have

1. $\tau(\Gamma) \geqslant \ell(\Gamma)\left(\frac{1}{12}\left(1-\frac{4}{\Lambda(\Gamma)}\right)^{2}+\frac{4(\Lambda(\Gamma)-2)}{(v+6) \Lambda(\Gamma)^{2}}\right)$, if $\Lambda(\Gamma) \geqslant 4$. In particular, $\tau(\Gamma) \geqslant \frac{\ell(\Gamma)}{108}$ if $\Lambda(\Gamma) \geqslant 6$, and $\tau(\Gamma) \geqslant \frac{\ell(\Gamma)}{300}$ if $\Lambda(\Gamma)=5$.

2. $\tau(\Gamma) \geqslant \frac{\ell(\Gamma)}{2(v+6)}$. In particular, $\tau(\Gamma) \geqslant \frac{\ell(\Gamma)}{108}$ if $v \leqslant 48$.

Proof. If an edge $e_{i} \in E(\Gamma)$ is a bridge of length $L_{i}$, then it contributes to $\tau(\Gamma)$ by $\frac{L_{i}}{4}$ (see [5, Corollaries 2.22 and 2.23] for more information). Therefore, we can assume that $\Gamma$ is bridgeless by using Remark 2.3. On the other hand, by using the scale-independence of the tau constant (see Remark 2.4), we can assume that $\Gamma$ is normalized. 
Now, we look for $x$ and $y$ values that satisfy the inequalities in parts (2), (3), and (4) of Theorem 6.9 and minimize $\frac{1}{12}-\frac{x}{6}+\frac{y}{6}$. This optimization problem can be solved by the following two ways using elementary calculus.

First way:

Figure 4 illustrates some of the inequalities given in Theorem 6.9. The lines $x+y=1$ and $x=3 y$ intersect at a point $B$ with coordinates $\left(\frac{3}{4}, \frac{1}{4}\right)$. Whenever $\Lambda(\Gamma) \geqslant 4$, we see that the line $x=(\Lambda(\Gamma)-1) y$ and the parabola $y=\frac{v+6}{4 v}(x+y)^{2}$ intersect at the point with coordinates $x=\frac{4 v(\Lambda(\Gamma)-1)}{(v+6) \Lambda(\Gamma)^{2}}$ and $y=\frac{4 v}{(v+6) \Lambda(\Gamma)^{2}}$, and that these give a lower bound to $\frac{1}{12}-\frac{x}{6}+\frac{y}{6}$. This proves the first inequality in part (1).

The line $\frac{1}{12}-\frac{x}{6}+\frac{y}{6}=c$ is tangential to the parabola $y=\frac{v+6}{4 v}(x+y)^{2}$ at the point with coordinates $x=\frac{3 v}{4(v+6)}$ and $y=\frac{v}{4(v+6)}$, and that these give a lower bound to $\frac{1}{12}-\frac{x}{6}+\frac{y}{6}$. This proves the first inequality in part (2).

The remaining parts are immediate from what we have shown.

Second way:

Using the inequalities in parts (3) and (4) of Theorem 6.9, we obtain $\sqrt{\frac{4 v}{v+6} y} \geqslant x+y \geqslant$ $\Lambda(\Gamma) y$ and $\sqrt{\frac{4 v}{v+6} y}-2 y \geqslant x-y$. Therefore, we obtain $y \leqslant \frac{4 v}{(v+6) \Lambda(\Gamma)^{2}}$ and $\tau(\Gamma)=\frac{1}{12}-\frac{x-y}{6} \geqslant$ $\frac{1}{12}-\frac{1}{6} \sqrt{\frac{4 v}{v+6} y}+\frac{y}{3}$. Since the minimum value of the parabola $f(y)=\frac{1}{12}-\frac{1}{6} \sqrt{\frac{4 v}{v+6} y}+\frac{y}{3}$ on $\left[0, \frac{4 v}{(v+6) \Lambda(\Gamma)^{2}}\right]$ occurs at $y=v /(4(6+v))$ for $\Lambda(\Gamma) \leqslant 3$, and at $y=\frac{4 v}{(v+6) \Lambda(\Gamma)^{2}}$ when $\Lambda(\Gamma) \geqslant 4$, one obtains the proof of the theorem.

Theorem 6.11. Let $\Gamma$ be a normalized bridgeless metrized graph. If all the edge lengths are equal to each other, then we have

$$
\frac{1}{12}-\frac{v-1}{6 e}+\frac{v-1}{3 e \Lambda(\Gamma)} \geqslant \tau(\Gamma) \geqslant \frac{1}{12}\left(\frac{g}{e}\right)^{2}+\frac{1}{2 v}\left(\frac{v-1}{e}\right)^{2} .
$$

In particular, if $\Gamma$ is an $n$-regular metrized graph and $\Lambda(\Gamma)=n$, we have

$$
\frac{1}{12}-\frac{(v-1)(n-2)}{3 v n^{2}} \geqslant \tau(\Gamma) \geqslant \frac{1}{12}-\frac{(v-1)\left((n-1) v^{2}-5 v+6\right)}{3 n^{2} v^{3}} .
$$

Proof. Since $L_{i}=\frac{1}{e}$ for each edge, $x(\Gamma)+y(\Gamma)=\frac{v-1}{e}$ by Equation (3) and Equation (21). Therefore, parts (3) and (4) of Theorem 6.9 are equivalent to $\frac{v-1}{\Lambda(\Gamma) e} \geqslant y \geqslant \frac{v+6}{4 v}\left(\frac{v-1}{e}\right)^{2}$, and Equation (20) is equivalent to $\tau(\Gamma)=\frac{1}{12}-\frac{v-1}{6 e}+\frac{y}{3}$. These give the first two inequalities. The final two inequalities follow from the fact that $e=\frac{n v}{2}$ when $\Gamma$ is $n$-regular.

\section{Cubic graphs}

In this section, we will show that Conjecture 1.1 holds for all metrized graphs if it holds for cubic metrized graphs. We call a 3-regular metrized graph a "cubic metrized graph" or "cubic graph" for short. We will consider the metrized graphs with $\kappa(\Gamma) \geqslant 2$ where 


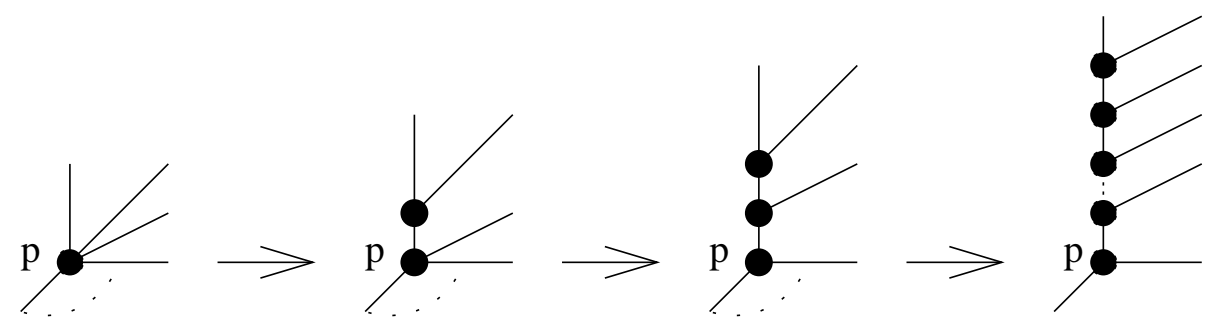

Figure 5: Transforming a non-cubic graph $\Gamma$ to a cubic graph.

$\kappa(\Gamma)$ is the vertex connectivity. By Remark 2.3, this would be enough to prove Conjecture 1.1 .

We will use the following notation and graph constructions.

Suppose $\Gamma$ is a normalized metrized graph, i.e., $\ell(\Gamma)=1$, and $p \in V(\Gamma)$ is a vertex with valence $n \geqslant 4$. We want to transform $\Gamma$ into another normalized metrized graph, $\Gamma_{p,(n-3)}^{N}$, by adding new edges and new vertices of valence 3 to $\Gamma$ in such a way that the valence of the vertex $p$ becomes 3 in $\Gamma_{p,(n-3)}^{N}$. In $\Gamma_{p,(n-3)}^{N}$, we add $n-3$ new vertices $p^{1}, p^{2}, \ldots, p^{n-3}$ and $n-$ 3 new edges $e_{p, 1}, e_{p, 2}, \ldots, e_{p,(n-3)}$ with pairs of end points $\left\{p^{1}, p^{2}\right\},\left\{p^{2}, p^{3}\right\}, \ldots,\left\{p^{n-3}, p\right\}$, respectively. Figure 5 shows the details of the transformation. The first graph in Figure 5 shows $\Gamma$.

Suppose the edges with end point $p$ are given in a specified order. We disconnect the first and the second edges from $p$. Then we reconnect them to $p$ via adding edge $e_{p, 1}$, with end points $\left\{p^{1}, p\right\}$ and of length $\varepsilon_{p, 1}$, so that the new vertex $p^{1}$ becomes the end point of the first edge, the second edge and the new edge $e_{p, 1}$. We denote this graph by $\Gamma_{p, 1}$. Note that $\ell\left(\Gamma_{p, 1}\right)=\ell(\Gamma)+\varepsilon_{p, 1}=1+\varepsilon_{p, 1}$ and if we contract the new edge $e_{p, 1}$, we obtain $\Gamma$. Also, the valence of $p$ in $\Gamma_{p, 1}$ is $n-1$. Then we obtain $\Gamma_{p, 1}^{N}$ by normalizing $\Gamma_{p, 1}$. $\Gamma_{p, 1}^{N}$ is the second graph in Figure 5 . Note that the graphs $\Gamma_{p, 1}$ and $\Gamma_{p, 1}^{N}$ have the same shape, i.e. the same topology. At the next step, we disconnect $e_{p, 1}$ and the third edge with vertex $p$ from $p$, then we reconnect them via adding the edge $e_{p, 2}$, with end points $\left\{p^{2}, p\right\}$ and of length $\varepsilon_{p, 2}$, so that the new vertex $p^{2}$ becomes the end point of third edge, $e_{p, 1}$ and $e_{p, 2}$. We denote this graph by $\Gamma_{p, 2}$. Note that the valence of $p$ in $\Gamma_{p, 2}$ is $n-2$. Then by normalizing $\Gamma_{p, 2}$ we obtain $\Gamma_{p, 2}^{N}$ which is shown by the third graph in Figure 5 . We continue this process until the valence of $p$ becomes 3 , i.e., until we obtain the graphs $\Gamma_{p,(n-3)}$ and $\Gamma_{p,(n-3)}^{N}$.

Note that $\varepsilon_{p, k}>0$ for each $k=1,2, \ldots, n-3$. Since $\kappa(\Gamma) \geqslant 2, \Gamma_{p, k}-e_{p, k}$ is connected for each $k=1,2, \ldots, n-3$. Let $\Gamma_{p, 0}^{N}:=\Gamma$.

Lemma 7.1. Let $k \in\{0,1, \ldots, n-4\}$ and let $\Gamma_{p, k+1}^{N}, \Gamma_{p, k}^{N}$, $p$ and $\varepsilon_{p, k+1}$ be as above. Then

$$
\tau\left(\Gamma_{p, k+1}^{N}\right) \leqslant \tau\left(\Gamma_{p, k}^{N}\right)+\frac{\varepsilon_{p, k+1}}{1+\varepsilon_{p, k+1}} \cdot\left(\frac{1}{12}-\tau\left(\Gamma_{p, k}^{N}\right)\right)
$$

Proof. Let $e_{p, k+1}, p^{k}, p^{k+1}, \Gamma, \Gamma_{p, k}^{N}, \Gamma_{p, k+1}, \Gamma_{p, k+1}^{N}, \varepsilon_{p, k+1}$ be as above.

Note that we can obtain $\Gamma_{p, k}^{N}$ from $\Gamma_{p, k+1}$ by contracting the edge $e_{p, k+1}$ to its end 
points. Since $\Gamma_{p, k}-e_{p, k}$ is connected, we can apply Lemma 3.1. This gives

$$
\tau\left(\Gamma_{p, k+1}\right)=\tau\left(\Gamma_{p, k}^{N}\right)+\frac{\varepsilon_{p, k+1}}{12}-\frac{\varepsilon_{p, k+1} A_{k}}{\bar{R}_{k+1}\left(\varepsilon_{p, k+1}+\bar{R}_{k+1}\right)},
$$

where $A_{k}:=A_{p^{k}, p^{k+1}, \Gamma_{p, k+1}-e_{p, k+1}}$ and $\bar{R}_{k+1}$ is the resistance, in $\Gamma_{p, k+1}-e_{p, k+1}$, between $p^{k}$ and $p^{k+1}$.

Since $\ell\left(\Gamma_{p, k+1}\right)=1+\varepsilon_{p, k+1}$,

$$
\tau\left(\Gamma_{p, k+1}\right)=\left(1+\varepsilon_{p, k+1}\right) \cdot \tau\left(\Gamma_{p, k+1}^{N}\right) .
$$

Substituting Equation (43) into Equation (42) gives

$$
\begin{aligned}
\tau\left(\Gamma_{p, k+1}^{N}\right) & =\frac{\tau\left(\Gamma_{p, k}^{N}\right)}{1+\varepsilon_{p, k+1}}+\frac{\varepsilon_{p, k+1}}{1+\varepsilon_{p, k+1}} \cdot\left(\frac{1}{12}-\frac{A_{k}}{\bar{R}_{k+1}\left(\varepsilon_{p, k+1}+\bar{R}_{k+1}\right)}\right) \\
& =\tau\left(\Gamma_{p, k}^{N}\right)+\frac{\varepsilon_{p, k+1}}{1+\varepsilon_{p, k+1}} \cdot\left(\frac{1}{12}-\frac{A_{k}}{\bar{R}_{k+1}\left(\varepsilon_{p, k+1}+\bar{R}_{k+1}\right)}-\tau\left(\Gamma_{p, k}^{N}\right)\right) \\
& \leqslant \tau\left(\Gamma_{p, k}^{N}\right)+\frac{\varepsilon_{p, k+1}}{1+\varepsilon_{p, k+1}} \cdot\left(\frac{1}{12}-\tau\left(\Gamma_{p, k}^{N}\right)\right),
\end{aligned}
$$

since $A_{k} \geqslant 0, \bar{R}_{k+1}>0$ and $\varepsilon_{p, k+1}>0$. This proves the result.

Theorem 7.2. If there exists a positive constant $C$ such that $\tau(\beta) \geqslant C$ for any normalized cubic graph $\beta$, then $\tau(\Gamma) \geqslant C$ for any normalized graph $\Gamma$.

Proof. Let $\Gamma$ be an arbitrary normalized metrized graph. By the additive property of the tau constant (Remark 2.3) we can assume that $\Gamma$ has no cut vertices. If $\Gamma$ is a loop, then $\tau(\Gamma)=\frac{1}{12}$. Thus, we can assume that $\Gamma$ has a vertex with valence at least 3 . After removing all vertices of valence 2 from $V(\Gamma)$, we can assume that all vertices have valence at least 3. Suppose $\Gamma$ is not a cubic graph. Then by basic graph theory $e>\frac{3}{2} v$, where $e=\#(E(\Gamma))$ and $v=\#(V(\Gamma))$ More precisely, this follows from the fact that the sum of the degrees of vertices equals twice the number of edges in any graph [11, pg 7 , Theorem 1.12]. Let $\varepsilon_{0}:=\frac{\varepsilon}{2 e-3 v}$, for some arbitrary $\varepsilon>0$.

Since $\Gamma$ is not cubic, there exists a vertex $p \in V(\Gamma)$ with $v(p) \geqslant 4$. We construct the graphs $\Gamma_{p, k+1}$ and $\Gamma_{p, k+1}^{N}$ for each $k=0,1, \ldots v(p)-4$ as mentioned at the beginning of this section. In these constructions, for each $k$ we take

$$
\varepsilon_{p, k+1}= \begin{cases}\frac{\varepsilon_{0}}{\frac{1}{12}-\tau\left(\Gamma_{p, k}^{N}\right)}, & \text { if } \frac{1}{12} \neq \tau\left(\Gamma_{p, k}^{N}\right) \\ \text { a positive number, } & \text { otherwise. }\end{cases}
$$

Note that $\frac{1}{12} \geqslant \tau\left(\Gamma_{p, k}^{N}\right)$ by [5, Corollary 5.8]. Then in both cases we have

$$
\tau\left(\Gamma_{p, k+1}^{N}\right) \leqslant \tau\left(\Gamma_{p, k}^{N}\right)+\varepsilon_{0}
$$


By considering Equation (45) for each $k=0,1, \ldots v(p)-4$, we obtain

$$
\tau\left(\Gamma_{p, v(p)-3}^{N}\right) \leqslant \tau(\Gamma)+(v(p)-3) \cdot \varepsilon_{0} .
$$

By following the same procedure for each $p \in V(\Gamma)$ with $v(p) \geqslant 4$, we obtain a normalized cubic graph $\beta$ such that

$$
\tau(\beta) \leqslant \tau(\Gamma)+\sum_{p \in V(\Gamma)}(v(p)-3) \cdot \varepsilon_{0}=\tau(\Gamma)+(2 e-3 v) \cdot \varepsilon_{0}=\tau(\Gamma)+\varepsilon .
$$

Thus $\tau(\Gamma) \geqslant C-\varepsilon$. Since $\varepsilon>0$ is arbitrary, $\tau(\Gamma) \geqslant C$.

Remark 7.3. Theorem 7.2 shows that to prove Conjecture 1.1, it is enough to establish it for cubic graphs.

Theorem 7.4. Let $\Gamma$ be a metrized graph with $\Lambda(\Gamma)=2$. Then there exists a metrized graph $\beta$ such that $\tau(\Gamma)=\tau(\beta), \ell(\Gamma)=\ell(\beta), \#(E(\Gamma)) \geqslant \#(E(\beta)), g(\Gamma)=g(\beta)$, and either $\Lambda(\beta) \geqslant 3$ or $\tau(\beta)=\frac{\ell(\beta)}{12}$.

Proof. Since $\Lambda(\Gamma)=2$, there is an edge $e_{i} \in E(\Gamma)$ such that $\Lambda\left(\Gamma-e_{i}\right)=1$, and let $L_{i}$ be the length of $e_{i}$. Let $C\left(e_{i}\right)=\left\{e_{i_{1}}, e_{i_{2}}, \ldots, e_{i_{s}}\right\}$ be the set of bridges in $\Gamma-e_{i}$, and let $L_{i_{j}}$ be the edge length of $e_{i_{j}}$ for each $1 \leqslant j \leqslant s$. Let $\gamma$ be the metrized graph obtained from $\Gamma$ by contracting all of the edges in $C\left(e_{i}\right)$ to their end points, and by extending the length $L_{i}$ of the edge $e_{i}$ to $L_{i}+\sum_{j=1}^{s} L_{i_{j}}$. We have $\ell(\Gamma)=\ell(\gamma)$, and $\tau\left(\Gamma-e_{i}\right)=\tau\left(\gamma-e_{i}\right)+\frac{1}{4} \sum_{j=1}^{s} L_{i_{j}}$ by additive property of the tau constant (see Remark 2.3), $R_{i}(\Gamma)=R_{i}(\gamma)+\sum_{j=1}^{s} L_{i_{j}}$ by elementary circuit reductions, and $L_{i}(\gamma)=L_{i}+\sum_{j=1}^{s} L_{i_{j}}$ by our construction. Moreover, $A_{p_{i}, q_{i}, \Gamma-e_{i}}=A_{p_{i}, q_{i}, \gamma-e_{i}}$ by the additive property of $A_{p, q, \Gamma}$ (see [5, Proposition 4.6]) and by $[5$, Proposition 4.5]. By our construction, $\#(E(\Gamma)) \geqslant \#(E(\gamma))$, and $g(\Gamma)=g(\gamma)$. If we apply Lemma 3.4 to $\tau(\Gamma)$ and $\tau(\gamma)$ and use the equalities we derived, we see that $\tau(\Gamma)=\tau(\gamma)$.

Note that either $\gamma$ has only one vertex in which case $\tau(\beta)=\frac{\ell(\beta)}{12}$ by [5, Proposition 2.28] (and $\Gamma$ is a cycle with possible loops) or $\Lambda\left(\gamma-e_{i}\right) \geqslant 2$. If $\gamma$ has a single vertex or $\Lambda(\gamma) \geqslant 3$, we take $\beta=\gamma$. Otherwise, $\Lambda(\gamma)=2$ in which case we can apply the same process to $\gamma$. We can repeat this process until we obtain a graph $\beta$ with the properties we wanted. Figure 6 shows an example in which this process applied four times.

Remark 7.5. One of the implications of Theorem 7.4 is that if Conjecture 1.1 holds for metrized graphs with edge connectivity at least 3, then it holds for all metrized graphs.

We show in $[6]$ that $\tau(\Gamma)$ can be computed by using the discrete Laplacian of $\Gamma$ and its pseudo inverse. In [4, Chapter 6], we constructed families of metrized graphs with the tau constants between $\frac{\ell(\Gamma)}{107}$ and $\frac{\ell(\Gamma)}{108}$, and the computations suggest that we can have sequences of metrized graphs with the tau constants approaching (but not equal) to $\frac{\ell(\Gamma)}{108}$. We recall that if Conjecture 1.1 holds with a constant $C$, then there is no metrized graph $\Gamma$ with $\tau(\Gamma)=C \cdot \ell(\Gamma)$ (see $[5$, Theorem 1.11]).

Based on our theoretical and computational investigations, we refine Conjecture 1.1 as follows:

Conjecture 7.6. For all metrized graphs $\Gamma, \tau(\Gamma)>\frac{\ell(\Gamma)}{108}$. 

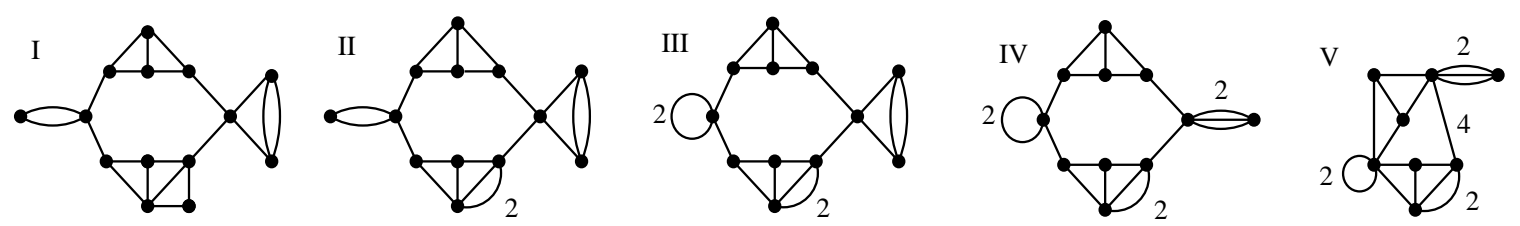

Figure 6: All of these graphs have equal tau constant. The last graph has edge connectivity 3 , and the others have edge connectivity 2 . The length of the extended edges are shown, the other edges have length 1.

\section{References}

[1] B. Bollabás, Extremal Graph Theory, Dover Publications Inc., Mineola, New York, 2004.

[2] M. Baker and X. Faber, Metrized graphs, Laplacian operators, and electrical networks, Quantum graphs and their applications, 15-33, Contemp. Math., 415, Amer. Math. Soc., Providence, RI, 2006.

[3] M. Baker and R. Rumely, Harmonic analysis on metrized graphs, Canad. J. Math: 59(2):225-275, 2005.

[4] Z. Cinkir, The Tau Constant of Metrized Graphs, Thesis at the University of Georgia, 2007.

[5] Z. Cinkir, The tau constant of a metrized graph and its behavior under graph operations, The Electronic Journal of Combinatorics, 18(1):P81, 2011.

[6] Z. Cinkir, The tau constant and the discrete Laplacian matrix of a metrized graph, European Journal of Combinatorics, 32(4):639-655, 2011.

[7] Z. Cinkir, Deletion and Contraction Identities for The Resistance Values and The Kirchhoff Index, International Journal of Quantum Chemistry, 111(15):4030-4041, 2011.

[8] Z. Cinkir, Zhang's Conjecture and The Effective Bogomolov Conjecture over Function Fields, Invent. Math., 183(3):517-562, 2011.

[9] T. Chinburg and R. Rumely, The capacity pairing, J. Reine Angew. Math. 434:1-44, 1993.

[10] Peter G. Doyle and J. Laurie Snell, Random Walks and Electrical Networks, Carus Math. Monogr., Mathematical Association of America, Washington D.C., 1984.

[11] R. Merris, Graph Theory, John Wiley \& Sons Inc., New York-Singapore-Toronto, 2001.

[12] R. Rumely, Capacity Theory on Algebraic Curves, Lecture Notes in Mathematics 1378, Springer-Verlag, Berlin-Heidelberg-New York, 1989.

[13] S. Zhang, Admissible pairing on a curve, Invent. Math., 112(1):171-193, 1993.

[14] S. Zhang, Gross-Schoen cycles and dualising sheaves, Invent. Math., 179(1):1-73, 2010. 